\title{
Normalized Study of Three-Parameter System in the Time Domain and Frequency Domain
}

\author{
Xiao-Lei Jiao, Yang Zhao, and Wen-Lai Ma \\ School of Astronautics, Harbin Institute of Technology, Harbin 150001, China \\ Correspondence should be addressed to Yang Zhao; yangzhao@hit.edu.cn
}

Received 12 April 2017; Revised 26 July 2017; Accepted 16 August 2017; Published 1 October 2017

Academic Editor: Naveed Ahmad

Copyright ( 2017 Xiao-Lei Jiao et al. This is an open access article distributed under the Creative Commons Attribution License, which permits unrestricted use, distribution, and reproduction in any medium, provided the original work is properly cited.

\begin{abstract}
Three-parameter isolation system can be used to isolate microvibration for control moment gyroscopes. Normalized analytical model for three-parameter system in the time domain and frequency domain is proposed by using analytical method. Dynamic behavior of three-parameter system in the time domain and frequency domain is studied. Response in the time domain under different types of excitations is analyzed. In this paper, a regulatory factor is defined in order to analyze dynamic behavior in the frequency domain. For harmonic excitation, a comparison study is made on isolation performance between the case when the system has optimal damping and the case when regulatory factor is 1. Besides, phase margin of three-parameter system is obtained. Results show that dynamic behavior in the time domain and frequency domain changes with regulatory factor. Phase margin has the largest value when the value of regulatory factor is 1 . System under impulse excitation and step excitation has the shortest settling time for the response in the time domain when the value of regulatory factor is 1 . When stiffness ratio is small, isolation performances of two cases are nearly the same; when system has a large stiffness ratio, isolation performance of the first case is better.
\end{abstract}

\section{Introduction}

The classic mass-spring-damper system includes spring and parallel viscous damper; it is two-parameter isolation system. Three-parameter system includes spring and an elastically supported damper. The system can be tuned by selecting parameter values that provide maximum damping at the fundamental frequency and reduced damping at higher frequencies [1]. Isolator based on three-parameter system is widely used in order to isolate microvibration in the aircraft. Microvibration is produced by control moment gyroscopes and reaction wheel assemblies with a frequency range of $0.1-300 \mathrm{~Hz}$. Microvibration in a satellite can take a long time to attenuate because of special space environment. It is necessary to protect high precision payloads from the influence of microvibration [2-12]. Isolator based on threeparameter system can be used to tackle this problem.

Early studies focused on two-parameter isolation system. References $[13,14]$ had a simple study on three-parameter system. They showed that the system had considerable advantages to prevent impact. Honeywell Inc. developed a series of isolators based on three-parameter system [1, 15-17]. The isolator had a pair of bellows which can be used to provide main stiffness; additional stiffness was volume stiffness produced by silicone oil. It was installed as a part of the complete reaction wheel isolation system; after being tested in Hubble Space Telescope, isolation performance was very good, with the maximum reduction in vibration, occurring at frequency as low as $50 \mathrm{~Hz}$, and the maximum attenuation rate was approximately $60 \mathrm{~dB}$. These isolators were assembled into isolation platform in order to prevent microvibration in all directions $[18,19]$. Then, a lot of isolators based on three-parameter system were developed [20-27]; isolation performance became better. Dynamic behavior of threeparameter system was studied by Brennan [28]; he pointed out that the system had critical damping if additional stiffness was at least eight times that of the main stiffness; the isolator afforded no advantages if the system was excited by white noise. Liu et al. [29] developed a fluid viscous damper based on three-parameter system; it was used to isolate vibration of the whole satellite; the performance was better than traditional isolator. Zhang et al. [30, 31] had a research on the performance of isolation platform based on three-parameter system. If parameters were selected reasonably, the system 


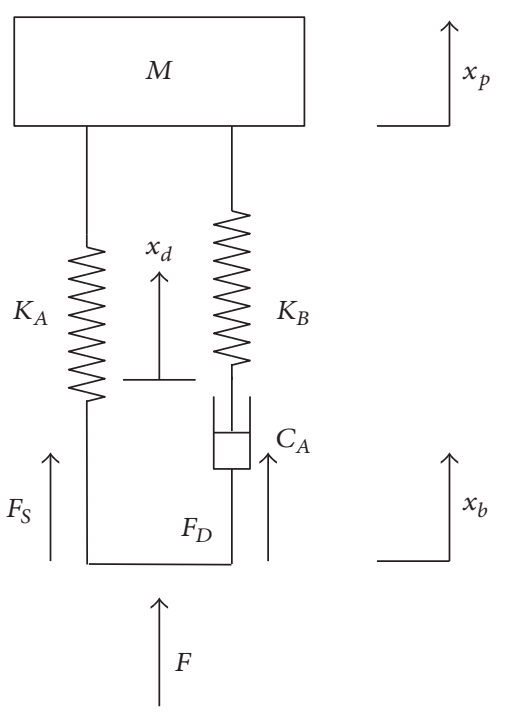

FIgURE 1: Three-parameter system (Zener model).

not only satisfied the requirement for vibration isolation but also guaranteed the stability of closed-loop attitude control system. Wang et al. [32,33] proposed a method to test stiffness and damping of the isolator based on threeparameter system; an equivalent two-parameter physical system was obtained based on mechanical impedance theory. Wang et al. [34] proposed a method to design isolator based on three-parameter system; optimal damping of the isolator was obtained. Wang et al. [35] had a research on nonlinear three-parameter system. An optimization method called the generalized pattern search (GPS) algorithm was proposed and applied to identify the nonlinear model parameters. Wang et al. [36] pointed out that the usage of nonlinear secondary spring had improved high frequency isolation performance and meanwhile maintained or even reduced its already low resonance amplitude. Shi et al. [37] developed a fluid viscous damper based on three-parameter system; they proposed equivalent stiffness and equivalent damping.

This study is concerned with dynamic behavior of threeparameter system in the time domain and frequency domain. By defining normalized parameters $\alpha$ and $\beta$, analytical normalized model of three-parameter system in the time domain and frequency domain is derived. After that, dynamic behavior in the frequency domain is obtained. System's response in the time domain under different types of excitation is analyzed. Then, we make a comparison research on isolation performance between the case when system has optimal damping and the case when the value of regulatory factor is 1. The results reveal that the second case has better isolation performance; this is a correction of previous studies.

\section{Normalized Model of Three-Parameter System}

2.1. Modeling of Three-Parameter System. Three-parameter system is shown in Figure 1. $M$ is mass of payload. $K_{A}$ is main stiffness of the system. $K_{B}$ is additional stiffness. $C_{A}$

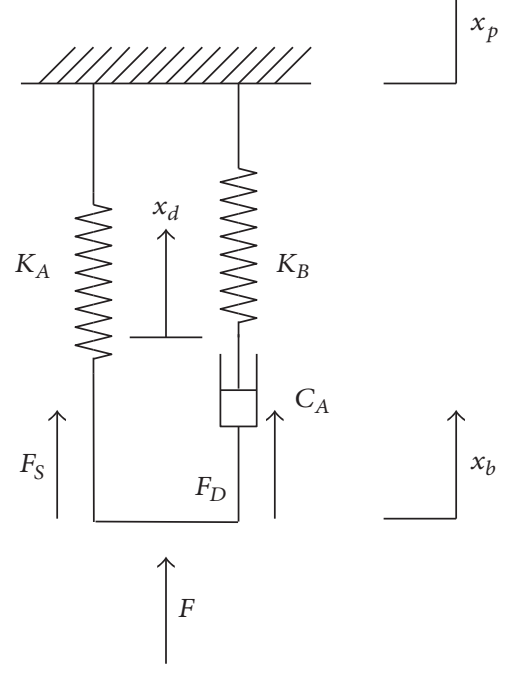

FIGURE 2: Mechanical impedance model.

is damping of the system. Figure 2 describes the mechanical impedance model of three-parameter system. $F$ is total force. $x_{p}$ is displacement of the payload. $x_{b}$ is displacement of the base. $x_{d}$ is displacement of damper node. $\dot{x}_{p}$ is velocity of the payload. $\dot{x}_{b}$ is velocity of the base. $\dot{x}_{d}$ is velocity of damper node.

The equations of motion for this system are

$$
\begin{aligned}
M \ddot{x}_{p}+K_{A}\left(x_{p}-x_{b}\right)+K_{B}\left(x_{p}-x_{d}\right) & =0, \\
K_{B}\left(x_{d}-x_{p}\right)+C_{A}\left(\dot{x}_{d}-\dot{x}_{b}\right) & =0 .
\end{aligned}
$$

Taking the Laplace transform and rearranging,

$$
\left[\begin{array}{cc}
\left(M s^{2}+K_{A}+K_{B}\right) & -K_{B} \\
-K_{B} & \left(K_{B}+C_{A} s\right)
\end{array}\right]\left[\begin{array}{l}
x_{p} \\
x_{d}
\end{array}\right]=\left[\begin{array}{c}
K_{A} \\
C_{A} s
\end{array}\right] x_{b} .
$$

$\delta$ is the stroke, $\delta=x_{b}-x_{p}, F_{S}$ is the static force, $F_{S}=K_{A} \delta$, $F_{D}$ is the dynamic force, $F_{D}=K_{B}\left(x_{d}-x_{p}\right)$, and $F$ is the total force, $F=F_{S}+F_{D}$.

2.2. Complex Mechanical Impedance. Figure 2 is schematic diagram of mechanical impedance model, one end of the model connected to the ground; therefore $x_{p}=0$.

The equations of motion for this system can be written as

$$
\begin{aligned}
& K_{A} x_{b}+C_{A}\left(\dot{x}_{b}-\dot{x}_{d}\right)=F, \\
& C_{A}\left(\dot{x}_{b}-\dot{x}_{d}\right)-K_{B} x_{d}=0 .
\end{aligned}
$$

Taking the Laplace transform and rearranging,

$$
\left[\begin{array}{cc}
\left(K_{A}+C_{A} s\right) & -C_{A} s \\
-C_{A} s & \left(K_{B}+C_{A} s\right)
\end{array}\right]\left[\begin{array}{l}
x_{b} \\
x_{d}
\end{array}\right]=\left[\begin{array}{l}
1 \\
0
\end{array}\right] F .
$$

The complex mechanical impedance $Z(s)$ can be written as

$$
Z(s)=\frac{K_{A} K_{B}+\left(K_{A}+K_{B}\right) C_{A} s}{K_{B}+C_{A} s} .
$$




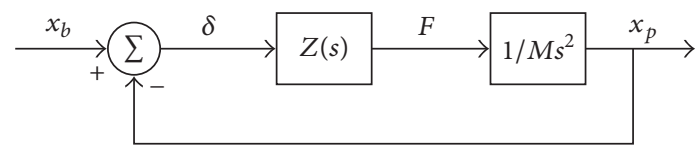

FIGURE 3: General isolator block diagram.

$\omega_{1}$ is zero (lead) frequency in impedance, $\omega_{1}=K_{A} K_{B} /\left(K_{A}+\right.$ $\left.K_{B}\right) C_{A} \cdot \omega_{2}$ is pole (lag) frequency in impedance, $\omega_{2}=K_{B} / C_{A}$.

Equation (5) becomes

$$
Z(s)=K_{A} \frac{\left(s / \omega_{1}+1\right)}{\left(s / \omega_{2}+1\right)} .
$$

For sinusoidal excitation, the complex mechanical impedance can be written as

$$
Z(\omega)=K_{A}\left[\frac{1+\omega^{2} / \omega_{1} \omega_{2}}{1+\omega^{2} / \omega_{2}^{2}}+\frac{\omega / \omega_{1}-\omega / \omega_{2}}{1+\omega^{2} / \omega_{2}^{2}}\right] .
$$

$\omega_{0}$ is maximum phase frequency, $\omega_{0}=\sqrt{\omega_{1} \omega_{2}}, \alpha$ is lead/lag separation ratio, $\alpha=\sqrt{\omega_{2} / \omega_{1}}, \Omega$ is normalized frequency, $\Omega=\omega / \omega_{0}$, transformation between $K_{A}, K_{B}, C_{A}$ and $K_{A}, \alpha$, $\omega_{0}$ can be written as

$$
\begin{aligned}
\alpha & =\sqrt{1+\frac{K_{B}}{K_{A}}}, \\
\omega_{0} & =\frac{K_{B}}{C_{A}} \sqrt{\frac{K_{A}}{K_{A}+K_{B}}}, \\
K_{B} & =K_{A}\left(1-\alpha^{2}\right), \\
C_{A} & =K_{A} \frac{\alpha^{2}-1}{\alpha \omega_{0}} .
\end{aligned}
$$

2.3. Normalized Open-Loop Transfer Function. Figure 3 is general isolator block diagram for three-parameter system; the open-loop transfer function can be written as

$$
G_{\mathrm{ol}}(s)=\frac{K_{A}}{M s^{2}} \frac{1+s / \omega_{1}}{1+s / \omega_{2}} .
$$

$G_{\mathrm{ol}}(s)$ is open-loop transfer function.

Figure 4 is Bode plot of the open-loop transfer function. Asymptotic gain is $K_{A} / M s^{2}$; the asymptote intersects with the lateral axis at frequency $\omega_{s}, \omega_{s}=\sqrt{K_{A} / M}$; (9) can be written as

$$
G_{\mathrm{ol}}(s)=\frac{\omega_{s}^{2}}{s^{2}} \frac{\left(1+\alpha\left(s / \omega_{0}\right)\right)}{\left(1+(1 / \alpha)\left(s / \omega_{0}\right)\right)} .
$$

$\omega_{s}^{2}=\left(\omega_{0} / \alpha\right) \omega_{\text {co }}, \omega_{\text {co }}$ is cross frequency in Bode plot of the open-loop transfer function, and it is marked in Figure $4 . \beta$ is regulatory factor, $\beta=\omega_{\mathrm{co}} / \omega_{0}$. $\omega_{s}$ can be written as $\omega_{s}=$ $\omega_{0} \sqrt{\beta / \alpha} ; G_{\mathrm{ol}}(s)$ can be written as

$$
G_{\mathrm{ol}}(s)=\frac{\beta}{\alpha} \frac{\omega_{0}^{2}}{s^{2}} \frac{\left(1+\alpha\left(s / \omega_{0}\right)\right)}{\left(1+(1 / \alpha)\left(s / \omega_{0}\right)\right)} .
$$

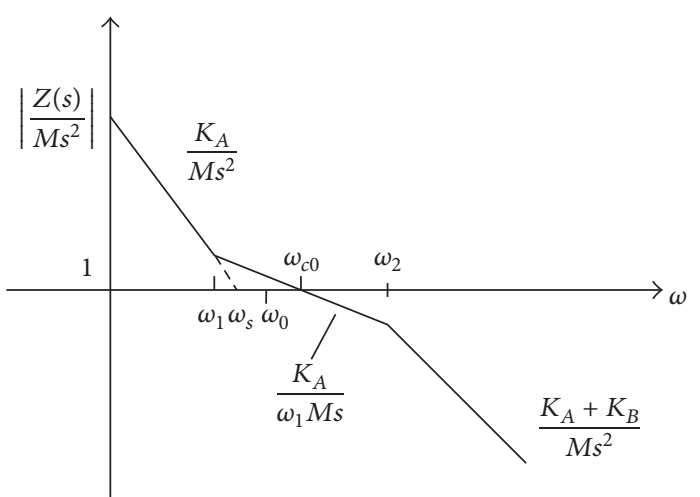

Figure 4: Bode plot of open-loop transfer function.

$\omega_{0}, \alpha, \beta$ can be written as

$$
\begin{aligned}
\omega_{0} & =\sqrt{\frac{K_{A} K_{B}^{2}}{\left(K_{A}+K_{B}\right) C_{A}^{2}}}, \\
\alpha & =\sqrt{\frac{K_{A}+K_{B}}{K_{A}}}, \\
\beta & =\frac{\left(K_{A}+K_{B}\right) C_{A}{ }^{2}}{M K_{B}{ }^{2}} \sqrt{\frac{K_{A}+K_{B}}{K_{A}}} .
\end{aligned}
$$

$K_{A}, K_{B}, C_{A}$ can be written as

$$
\begin{aligned}
& K_{A}=M \omega_{0}^{2} \frac{\beta}{\alpha}, \\
& K_{B}=M \omega_{0}^{2}\left(\alpha^{2}-1\right) \frac{\beta}{\alpha}, \\
& C_{A}=M \omega_{0}\left(\alpha^{2}-1\right) \frac{\beta}{\alpha^{2}} .
\end{aligned}
$$

When $\beta=1, K_{A}, K_{B}, C_{A}$ can be written as

$$
\begin{aligned}
K_{A} & =\frac{M \omega_{0}^{2}}{\alpha} \\
K_{B} & =M \omega_{0}^{2} \frac{\left(\alpha^{2}-1\right)}{\alpha}, \\
C_{A} & =\frac{M \omega_{0}\left(\alpha^{2}-1\right)}{\alpha^{2}} .
\end{aligned}
$$

If parameters of physical model $\left(K_{A}, K_{B}, C_{A}\right)$ or normalized parameters $(\alpha, \beta)$ are known, transmissibility curve can be obtained; it is convenient to design three-parameter system if we use normalized model.

2.4. Normalized Close-Loop Transfer Function. According to block diagram of open-loop transfer function, isolation transfer function can be written as

$$
G_{x p \_x b}(s)=\frac{x_{p}(s)}{x_{b}(s)} .
$$


$G_{x p \_x b}(s)$ is open-loop transfer function. Equation (15) can be written as

$$
G_{x p \_x b}(s)=\frac{G_{\mathrm{ol}}(s)}{1+G_{\mathrm{ol}}(s)} .
$$

Defining $\psi=s / \omega_{0},(16)$ can be written as

$$
G_{x p-x b}(\psi)=\frac{C(\psi)}{R(\psi)}=\frac{1+\alpha \sqrt{\beta} \psi}{1+\alpha \sqrt{\beta} \psi+\alpha \psi^{2}+\sqrt{\beta} \psi^{3}} .
$$

$C(\psi)$ is numerator, $C(\psi)=1+\alpha \sqrt{\beta} \psi$, and $R(\psi)$ is denominator, $R(\psi)=1+\alpha \sqrt{\beta} \psi+\alpha \psi^{2}+\sqrt{\beta} \psi^{3}$.

Equation (17) can be written as

$$
\begin{aligned}
& G_{x p-x b}(s) \\
& \quad=\frac{1+\left(\alpha \sqrt{\beta} / \omega_{0}\right) s}{1+\left(\alpha \sqrt{\beta} / \omega_{0}\right) s+\left(\alpha / \omega_{0}^{2}\right) s^{2}+\left(\sqrt{\beta} / \omega_{0}^{3}\right) s^{3}} .
\end{aligned}
$$

When $\beta=1$, (17) can be written as

$$
G_{x p \_x b}(\psi)=\frac{C(\psi)}{R(\psi)}=\frac{1+\alpha \psi}{1+\alpha \psi+\alpha \psi^{2}+\psi^{3}} .
$$

\section{Dynamic Behavior in the Frequency Domain}

3.1. Root Locus. According to (17), characteristic equation can be written as

$$
\begin{aligned}
1+\alpha \sqrt{\beta} \psi+\alpha \psi^{2}+\sqrt{\beta} \psi^{3}= & 0, \\
& \left(\alpha=\sqrt{\frac{K_{A}+K_{B}}{K_{A}}}>1\right) .
\end{aligned}
$$

The shape of root locus will be diverse because of different values of $\alpha$.

Equation (20) can be written as

$$
\frac{1}{\sqrt{\beta}} \frac{1+\alpha \psi^{2}}{\psi^{3}+\alpha \psi}=-1
$$

Figure 5 is root locus for $\alpha<3$. Figure 6 is root locus for $\alpha=3$. Figure 7 is root locus for $\alpha>3$. Root locus is located on the left side of lateral axis; it means that the system is stable. Each root locus has three branches corresponding to three characteristic roots.

(1) If $\alpha<3$, the system has real poles and conjugate poles. When the system has real poles, it means that the system has critical damping. Free vibration mode of the system is shown in Figure 8 (red line), which has obvious oscillation.

(2) If $\alpha=3$, root locus intersects with lateral axis at point $(-1,0)$. Real poles mean that the system has critical damping. Free vibration mode is shown in Figure 8 (green line); the oscillation is not obvious.

(3) If $\alpha>3$, the system has real poles and conjugate poles. Real poles mean that the system has critical damping. Free

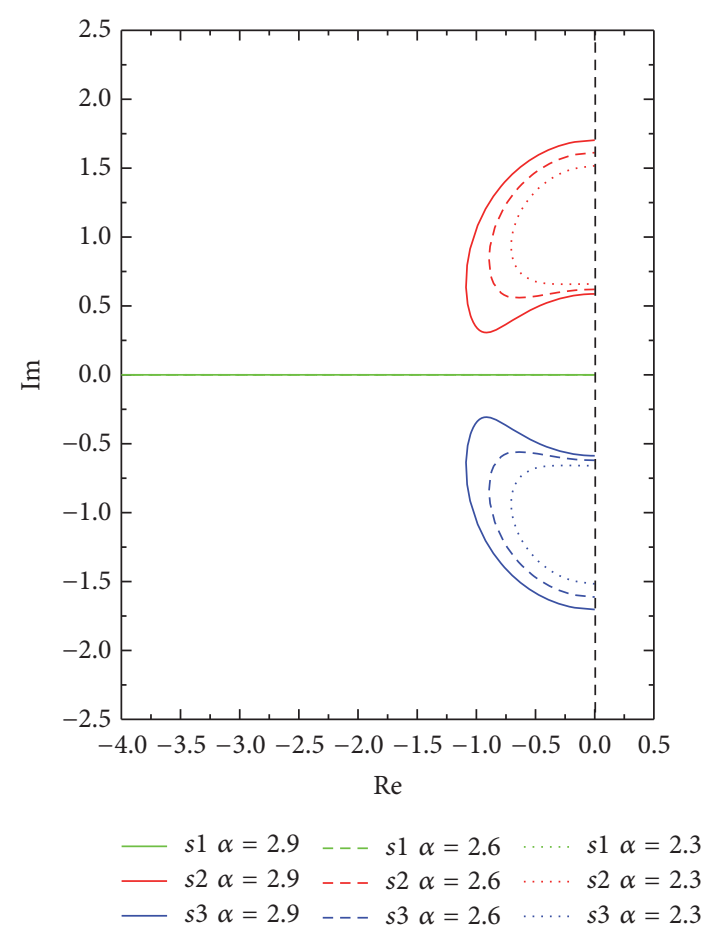

Figure 5: Root locus $(\alpha<3)$.

vibration mode of the system is shown in Figure 8 (blue line); oscillation decays with time quickly.

When $\beta=1$, denominator of close-loop transfer function can be written as

$$
R(\psi)=\psi^{3}+\alpha \psi^{2}+a \psi+1
$$

Equation (22) can be written as

$$
R(\psi)=(\psi+1)\left[\psi^{2}+\psi(\alpha-1)+1\right] .
$$

For $\beta=1$, free vibration mode of the system will be analyzed for different values of $\alpha$.

(1) If $\alpha>3, \Delta>0$, and $\Delta=(\alpha-1)^{2}-4$, there are three different real poles

$$
\begin{aligned}
& r_{1}=-1, \\
& r_{2}=\frac{-(\alpha-1)+\sqrt{(\alpha-1)^{2}-4}}{2}, \\
& r_{3}=\frac{-(\alpha-1)-\sqrt{(\alpha-1)^{2}-4}}{2} .
\end{aligned}
$$

These three real poles mean that the system has three different free vibration modes

$$
\begin{aligned}
& c_{1}(t)=e^{-t}, \\
& c_{2}(t)=e^{\left(\left(-(\alpha-1)+\sqrt{(\alpha-1)^{2}-4}\right) / 2\right) t}, \\
& c_{3}(t)=e^{\left(\left(-(\alpha-1)-\sqrt{(\alpha-1)^{2}-4}\right) / 2\right) t} .
\end{aligned}
$$




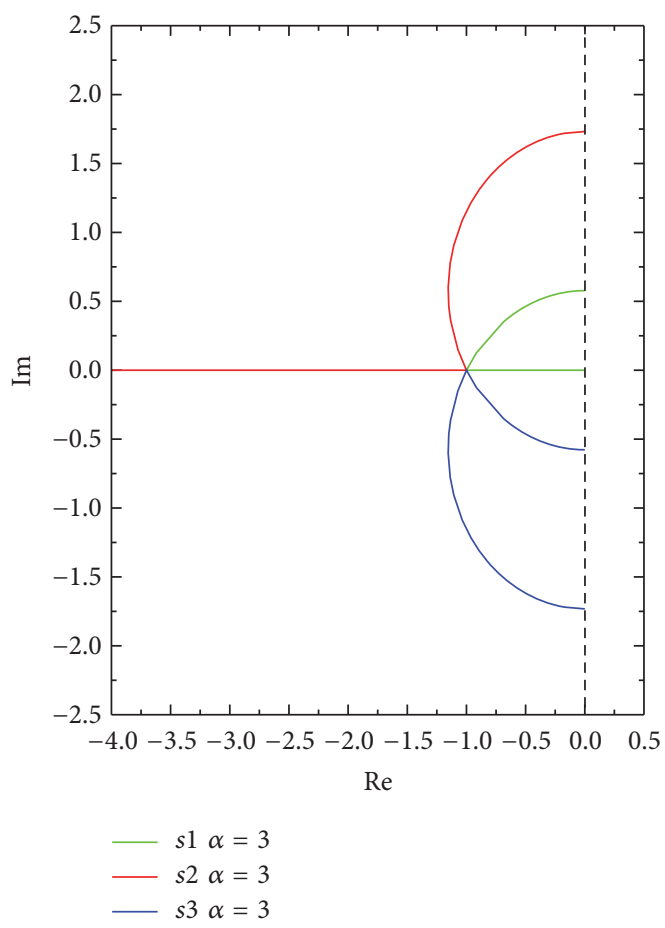

Figure 6: Root locus $(\alpha=3)$.

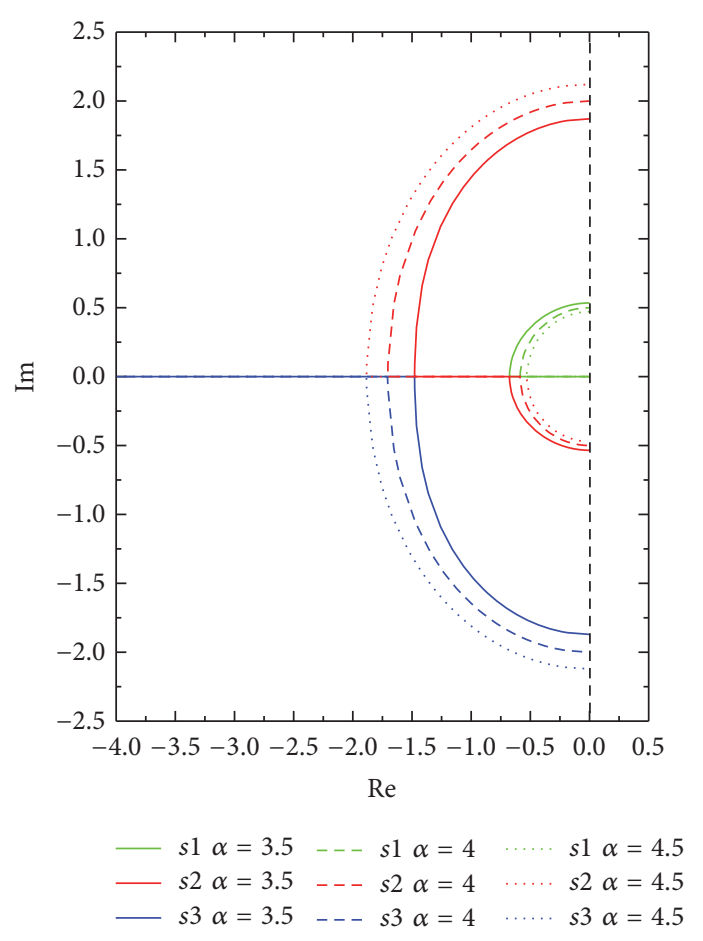

Figure 7: Root locus $(\alpha>3)$. 


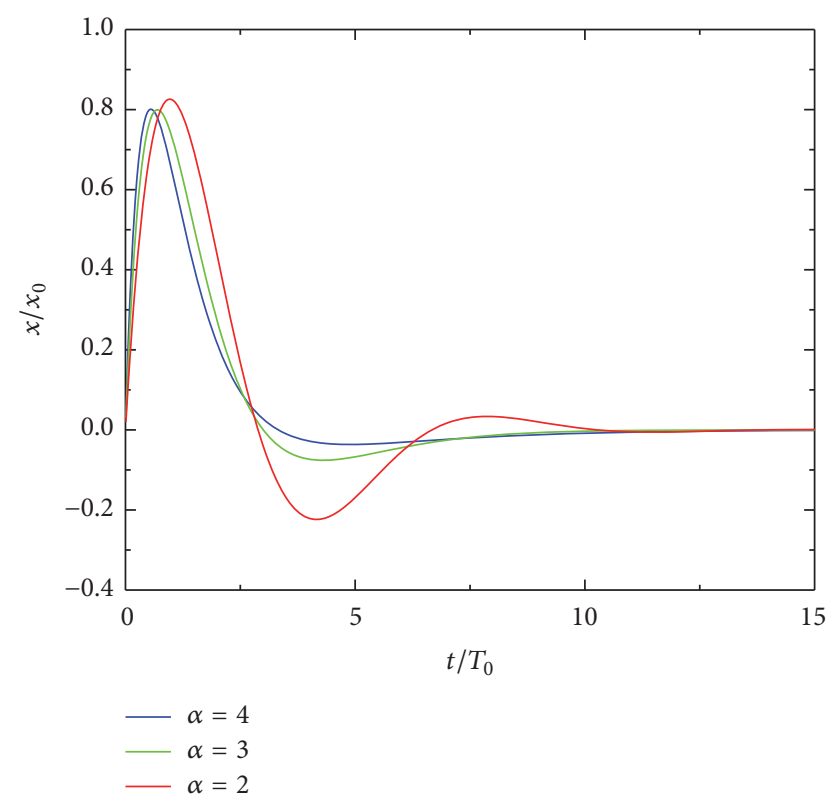

FIGURE 8: Free motion modes for different values of $\alpha$.

For unit pulse excitation, response in the time domain can be written as

$$
\begin{aligned}
c(t) & =\alpha\left[\frac{1 / \alpha+r_{1}}{\left(r_{1}-r_{2}\right)\left(r_{1}-r_{3}\right)} e^{r_{1} t}\right. \\
& \left.+\frac{1 / \alpha+r_{2}}{\left(r_{2}-r_{1}\right)\left(r_{2}-r_{3}\right)} e^{r_{2} t}+\frac{1 / \alpha+r_{3}}{\left(r_{3}-r_{1}\right)\left(r_{3}-r_{2}\right)} e^{r_{3} t}\right] .
\end{aligned}
$$

(2) If $\alpha=3$ and $\Delta=0$, there is a single real pole

$$
r=-1
$$

$$
\begin{aligned}
c(t) & =\alpha\left\{\frac{1 / \alpha-1}{((\alpha-1) / 2-1)^{2}+1-((\alpha-1) / 2)^{2}} e^{-t}\right. \\
& +\frac{1}{\sqrt{1-((\alpha-1) / 2)^{2}}}\left[\frac{(1 / \alpha-(\alpha-1) / 2)^{2}+1-((\alpha-1) / 2)^{2}}{(1-(\alpha-1) / 2)^{2}+1-((\alpha-1) / 2)^{2}}\right]^{1 / 2} e^{-((\alpha-1) / 2) t} \sin \left[\sqrt{\left.\left.1-\left(\frac{\alpha-1}{2}\right)^{2} t+\varphi\right]\right\} .}\right.
\end{aligned}
$$

$\varphi$ is phase angle.

According to (23), zero-pole plots for different values of $\alpha$ can be obtained.

Figures 9, 10, and 11 are zero-pole plots for different values of $\alpha$.

If $\alpha=2$, there is a real pole and a pair of conjugate poles. The real pole means that the system has critical damping. It can be seen from Figure 9 that the system has dominant poles; it means that free vibration modes corresponding to conjugate poles are main modes. If $\alpha=3$, there is a real pole and a pair of conjugate poles; it can be seen from Figure 10
It means that the system has a single free vibration mode; for unit pulse excitation, response in the time domain can be written as

$$
c(t)=\alpha\left[\frac{1-\alpha}{2} t^{2}+\alpha t\right] e^{-t}
$$

(3) If $1<\alpha<3$ and $\Delta<0$, there is a real pole and a pair of conjugate poles

$$
\begin{aligned}
& r_{1}=-1, \\
& r_{2}=\frac{-(\alpha-1)+j \sqrt{4-(\alpha-1)^{2}}}{2}, \\
& r_{3}=\frac{-(\alpha-1)-j \sqrt{4-(\alpha-1)^{2}}}{2} .
\end{aligned}
$$

Free vibration modes can be written as

$$
\begin{aligned}
& c_{1}(t)=e^{-t}, \\
& c_{2}(t)=e^{-((\alpha-1) / 2) t}\left(\cos \frac{\sqrt{(\alpha-1)^{2}-4}}{2} t\right. \\
& \left.+\sin \frac{\sqrt{(\alpha-1)^{2}-4}}{2} t\right) .
\end{aligned}
$$

For unit pulse excitation, response in the time domain can be written as

that imaginary part of conjugate poles is very small. If $\alpha=4$, there are three different real poles which means the system has critical damping.

3.2. Nyquist Diagram. Open-loop transfer function can be written as

$$
G_{\mathrm{ol}}(\psi)=\frac{1+\alpha \sqrt{\beta} \psi}{\sqrt{\beta} \psi^{3}+\alpha \psi^{2}} .
$$




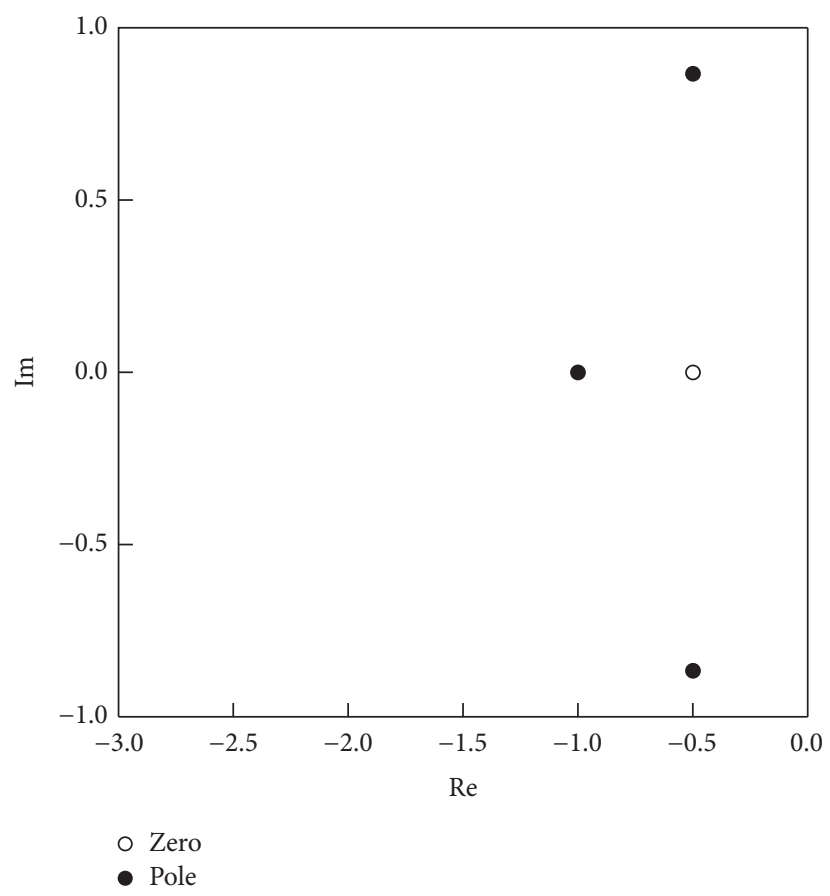

Figure 9: Zero-pole plot $(\alpha=2, \beta=1)$.

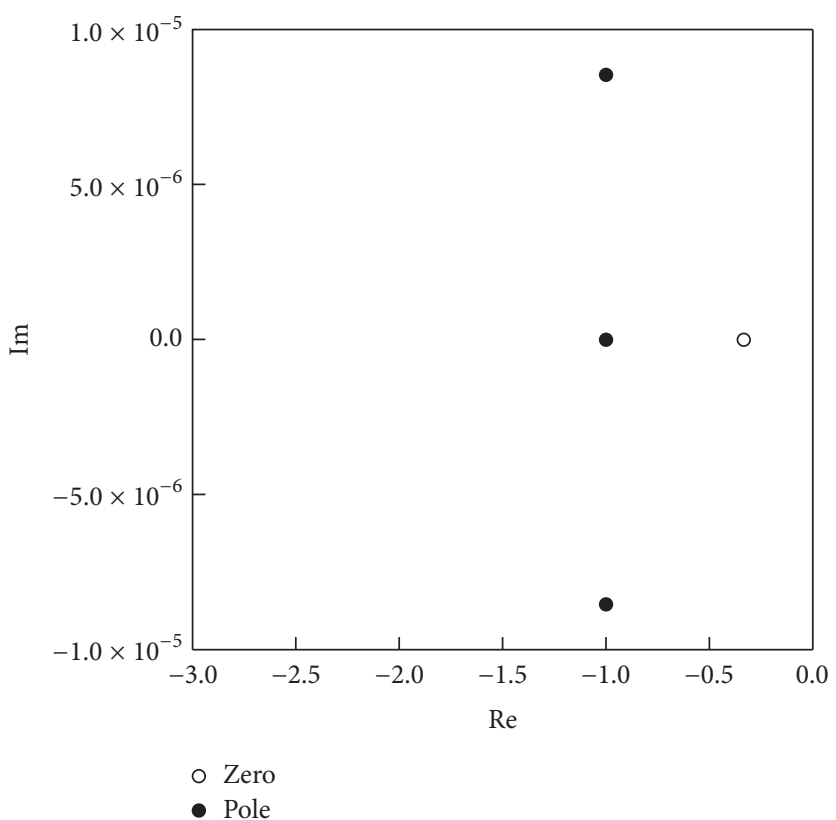

Figure 10: Zero-pole plot $(\alpha=3, \beta=1)$.

Nyquist diagram can be obtained according to the open-loop transfer function.

Figures 12, 13, 14, and 15 are the Nyquist diagrams for different values of $\alpha$. It can be concluded from Nyquist diagram that phase margin has the maximum value when $\beta=1$; phase margin increases with the value of $\alpha$, which means that stability of system increases too.
3.3. Amplitude-Frequency Characteristic and Phase-Frequency Characteristic. Transmissibility is an important index for isolation performance of the isolator. In this section, amplitudefrequency characteristic and phase-frequency characteristic of three-parameter system will be analyzed.

Figures 16-19 have described three-parameter system's amplitude-frequency characteristic and phase-frequency 


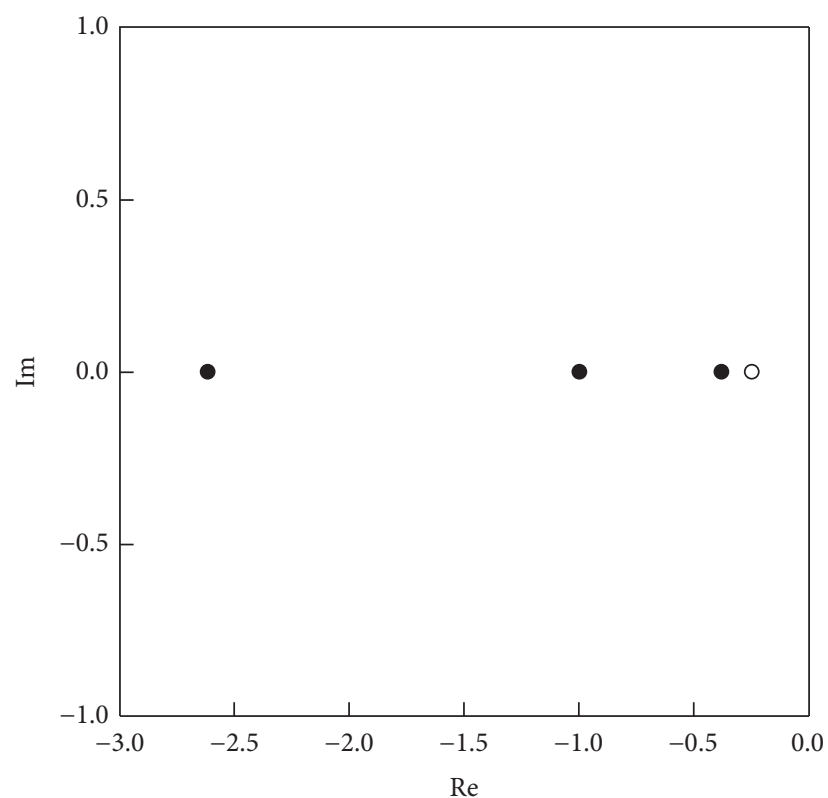

○ Zero

- Pole

Figure 11: Zero-pole plot $(\alpha=4, \beta=1)$.

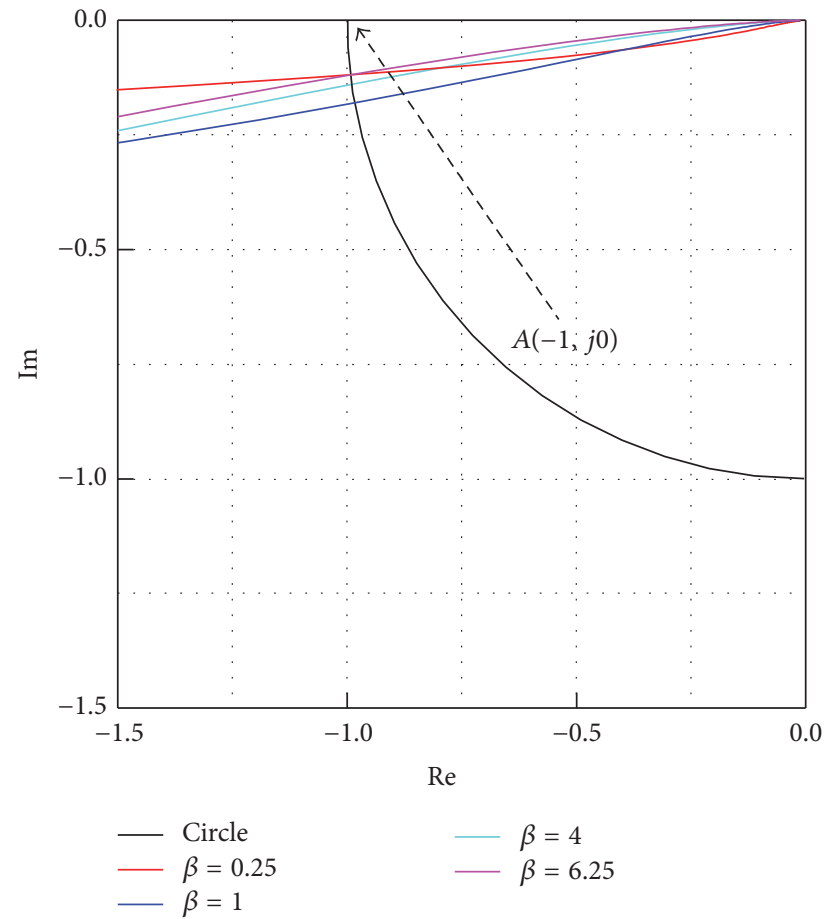

Figure 12: Nyquist diagram $(\alpha=1.2)$.

characteristic for different values of $\alpha$ and $\beta$. It can be concluded that when $\beta=1$, system has a minimum resonance peak compared with the case when $\beta<1$ and $\beta>1$; resonance peak decreases with the value of $\alpha$. The case when $\beta=1$ is very important; it can be used to design an isolator which has minimum resonance peak.

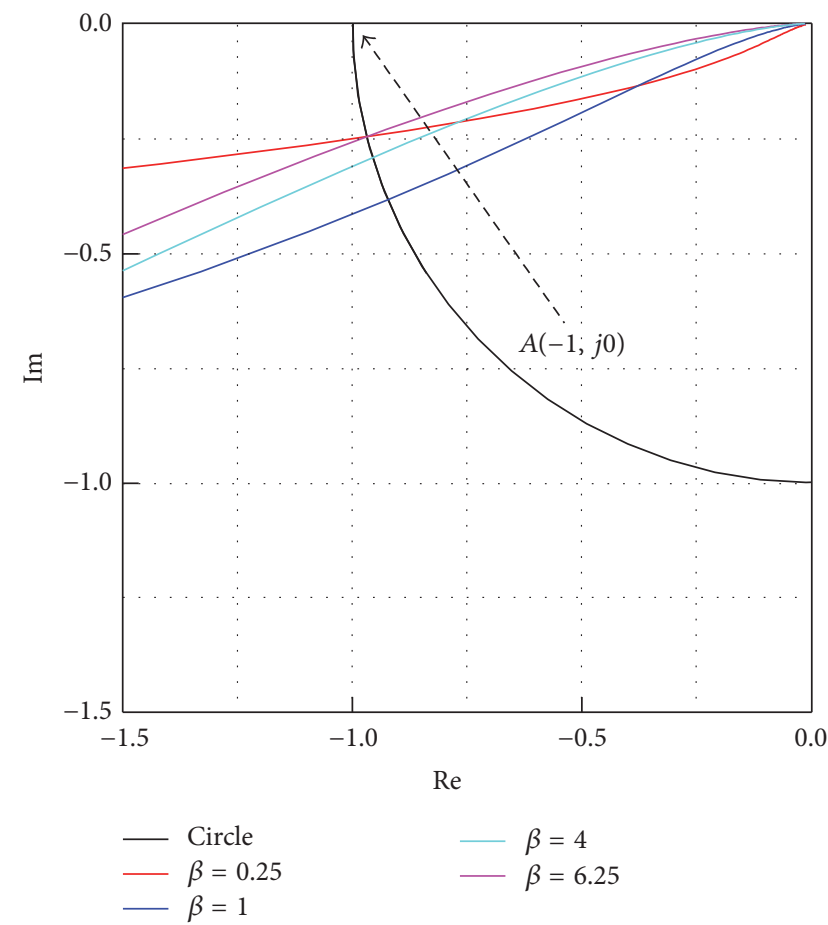

Figure 13: Nyquist diagram $(\alpha=1.5)$.

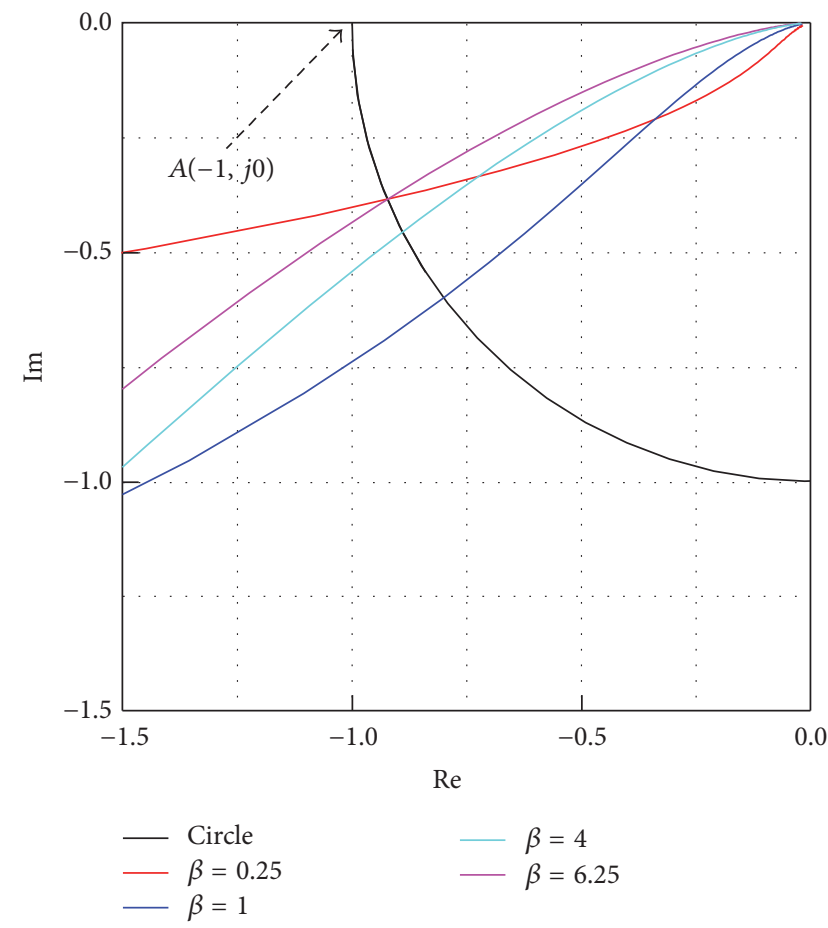

FIgURE 14: Nyquist diagram $(\alpha=2)$.

\section{Dynamic Behavior in the Time Domain}

4.1. Response in the Time Domain under Different Types of Excitations. When system is applied to different types of excitation, response in the time domain will be different. In 


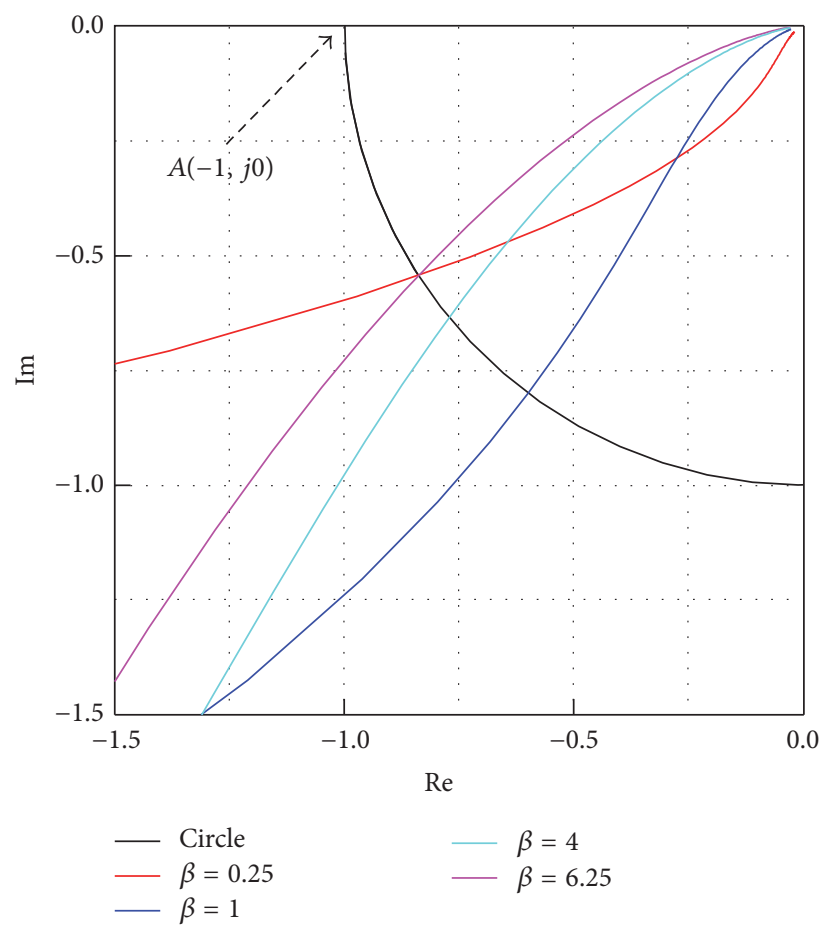

FIGURE 15: Nyquist diagram $(\alpha=3)$.
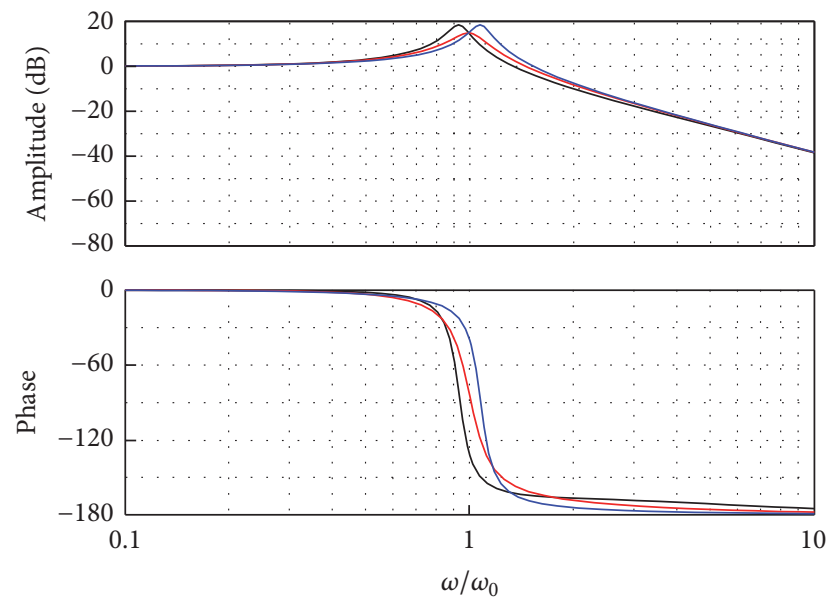

$$
\begin{aligned}
\alpha & =1.2, \beta=0.16 \\
-\alpha & =1.2, \beta=1 \\
-\alpha & =1.2, \beta=6.25
\end{aligned}
$$

FIGURE 16: Amplitude-frequency characteristic and phase-frequency characteristic $(\alpha=1.2, \beta=0.16,1,6.25)$.

this section, response in the time domain for different types of excitation will be analyzed.

(1) Step Excitation. As has been analyzed in Section 3.1, characteristic root has relationship with the value of $\Delta$. In this section, only the case when system has a real root and conjugate roots will be analyzed.
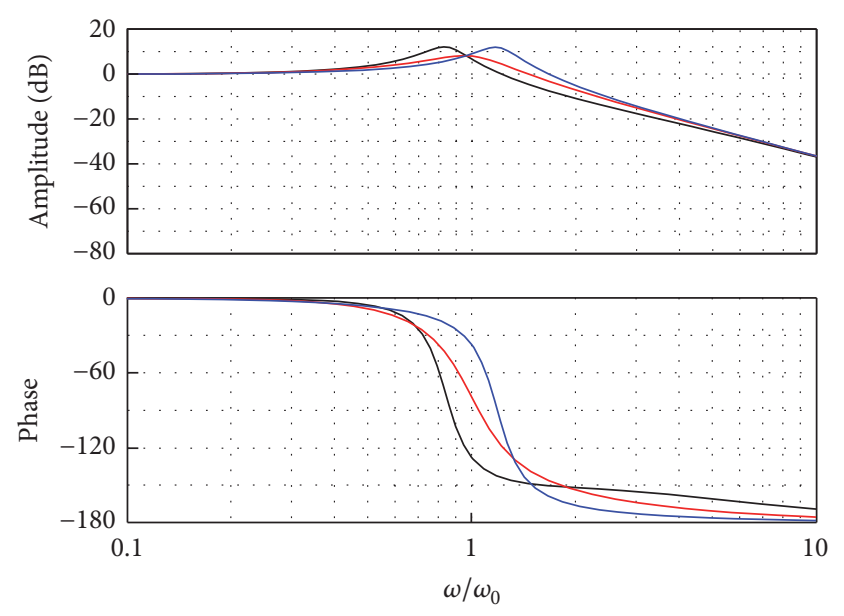

$$
\begin{aligned}
\alpha & =1.5, \beta=0.16 \\
-\alpha & =1.5, \beta=1 \\
\alpha & =1.5, \beta=6.25
\end{aligned}
$$

FIgURE 17: Amplitude-frequency characteristic and phase-frequency characteristic $(\alpha=1.5, \beta=0.16,1,6.25)$.

For step excitation, output function can be written as

$$
\begin{aligned}
& C(\psi)=\frac{1}{\sqrt{\beta}} \frac{\alpha \sqrt{\beta} \psi+1}{\psi\left(\psi-\psi_{1}\right)\left(\psi-\psi_{2}\right)\left(\psi-\psi_{3}\right)}, \\
& \qquad\left(\psi=\frac{s}{\omega_{0}}\right) .
\end{aligned}
$$

Equation (33) has three characteristic roots $\psi_{1}, \psi_{2}, \psi_{3}$ which can be written as

$$
\begin{aligned}
& \psi_{1}=c, \\
& \psi_{2}=a+b i, \\
& \psi_{3}=a-b i .
\end{aligned}
$$

$c$ is real root, $a$ is real part of complex root, and $b$ is the imaginary part of complex root. Response in the time domain can be derived by using inverse Laplace transform method; it can be written as

$c(t)$

$$
=\frac{1}{\sqrt{\beta}}\left(A_{0}+A_{1} e^{c t}+2 U e^{a t} \cos b t-2 V e^{a t} \sin b t\right),
$$

$$
\begin{aligned}
& A_{0}=-\frac{1}{c\left(a^{2}+b^{2}\right)}, \\
& A_{1}=\frac{1 / c+\alpha \sqrt{\beta}}{\left(c^{2}-a^{2}\right)+b^{2}}, \\
& U=\frac{p_{1} p_{3}+p_{2} p_{4}}{p_{3}^{2}+p_{4}^{2}}, \\
& V=\frac{p_{1} p_{4}-p_{2} p_{3}}{p_{3}{ }^{2}+p_{4}{ }^{2}},
\end{aligned}
$$



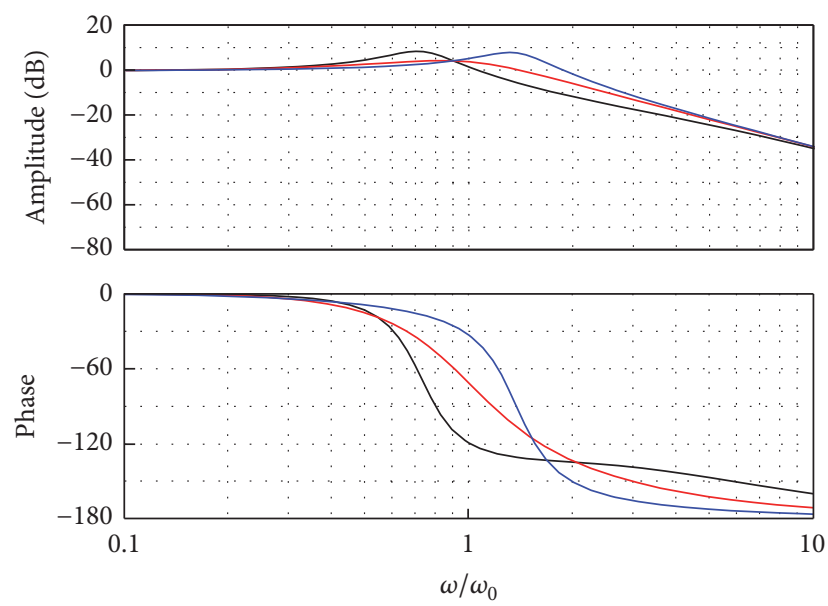

$$
\begin{aligned}
\alpha & =2, \beta=0.16 \\
-\alpha & =2, \beta=1 \\
\alpha & =2, \beta=6.25
\end{aligned}
$$

Figure 18: Amplitude-frequency characteristic and phase-frequency characteristic $(\alpha=2, \beta=0.16,1,6.25)$.

$$
\begin{aligned}
& p_{1}=m a+1, \\
& p_{2}=m b, \\
& p_{3}=-4 a b^{2}+2 c b^{2}, \\
& p_{4}=2 a b(a-c)-2 b^{3}, \\
& m=\alpha \sqrt{\beta} .
\end{aligned}
$$

(2) Slope Excitation. For slope excitation, output function can be written as

$$
C(\psi)=\frac{1}{\sqrt{\beta}} \frac{\alpha \sqrt{\beta} \psi+1}{\psi^{2}\left(\psi-\psi_{1}\right)\left(\psi-\psi_{2}\right)\left(\psi-\psi_{3}\right)} .
$$

Three characteristic roots are $\psi_{1}, \psi_{2}, \psi_{3}$, which can be written as

$$
\begin{aligned}
& \psi_{1}=c, \\
& \psi_{2}=a+b i, \\
& \psi_{3}=a-b i .
\end{aligned}
$$

$c$ is real root, $a$ is real part of complex root, and $b$ is the imaginary part of complex root. Response in the time domain can be derived by using inverse Laplace transform method; it can be written as

$$
\begin{aligned}
c(t) & =\frac{1}{\sqrt{\beta}}\left\{A_{0} t+A_{1}+A_{2} e^{-c t}\right. \\
+ & \left.2 e^{a t}(-F \sin b t+E \cos b t)\right\}, \\
A_{0} & =-\frac{1}{c\left(a^{2}+b^{2}\right)}
\end{aligned}
$$
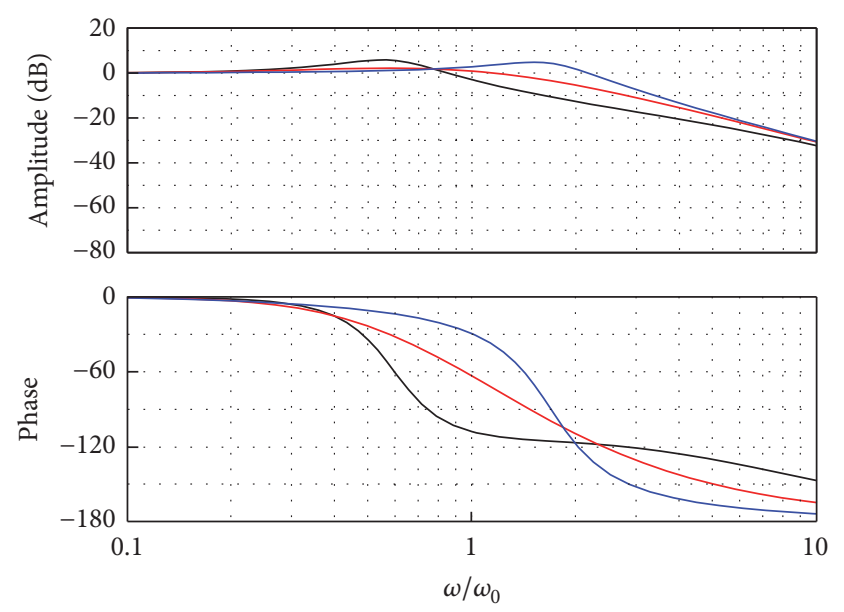

$$
\begin{aligned}
\alpha & =3, \beta=0.16 \\
\alpha & =3, \beta=1 \\
\alpha & =3, \beta=6.25
\end{aligned}
$$

FIgURE 19: Amplitude-frequency characteristic and phase-frequency characteristic $(\alpha=3, \beta=0.16,1,6.25)$.

$$
\begin{aligned}
& A_{1}=\frac{-m c\left(a^{2}+b^{2}\right)-\left(a^{2}+b^{2}+2 a c\right)}{c^{2}\left(a^{2}+b^{2}\right)^{2}}, \\
& A_{2}=\frac{m c+1}{c^{2}\left[(c-a)^{2}+b^{2}\right]}, \\
& p_{1}=m a+1, \\
& p_{2}=m b, \\
& p_{3}=-2 b^{2}\left(a^{2}-b^{2}\right)-4 a b^{2}(a-c), \\
& p_{4}=2 b\left(a^{2}-b^{2}\right)(a-c)-4 a b^{3}, \\
& E=\frac{p_{1} p_{3}+p_{2} p_{4}}{p_{3}^{2}+p_{4}^{2}}, \\
& F=\frac{p_{2} p_{3}-p_{1} p_{4}}{p_{3}^{2}+p_{4}^{2}}, \\
& m=\alpha \sqrt{\beta} .
\end{aligned}
$$

(3) Acceleration Excitation. For acceleration excitation, output function can be written as

$$
C(\psi)=\frac{1}{\sqrt{\beta}} \frac{\alpha \sqrt{\beta} \psi+1}{\psi^{3}\left(\psi-\psi_{1}\right)\left(\psi-\psi_{2}\right)\left(\psi-\psi_{3}\right)} .
$$

Three characteristic roots are $\psi_{1}, \psi_{2}, \psi_{3}$, which can be written as

$$
\begin{aligned}
& \psi_{1}=c \\
& \psi_{2}=a+b i \\
& \psi_{3}=a-b i
\end{aligned}
$$


$c$ is real root, $a$ is real part of complex root, and $b$ is the imaginary part of complex root.
Response in the time domain can be derived by using inverse Laplace transform method; it can be written as

$$
\begin{aligned}
c(t) & =\frac{1}{\sqrt{\beta}}\left[\frac{1}{2} A_{0} t^{2}+A_{1} t+A_{2}+A_{3} e^{c t}+2 e^{a t}(-F \sin b t+E \cos b t)\right], \\
A_{0} & =-\frac{1}{c\left(a^{2}+b^{2}\right)}, \\
A_{1} & =\frac{-m c\left(a^{2}+b^{2}\right)-\left(a^{2}+b^{2}+2 a c\right)}{c^{2}\left(a^{2}+b^{2}\right)^{2}}, \\
A_{2} & =\frac{(c+2 a)\left[-c\left(a^{2}+b^{2}\right)\right]-\left(a^{2}+b^{2}+2 a c\right)\left\{m\left[-c\left(a^{2}+b^{2}\right)\right]-\left(a^{2}+b^{2}+2 a c\right)\right\}}{\left[-c\left(a^{2}+b^{2}\right)\right]^{3}}, \\
E & =\frac{p_{1} p_{3}+p_{2} p_{4}}{p_{3}^{2}+p_{4}^{2}}, \\
p_{4} & =\frac{p_{2} p_{3}-p_{1} p_{4}}{p_{3}^{2}+p_{4}^{2}}, \\
p_{3} & =b\left(3 a^{2}-b^{2}\right)+a(a-c)\left(3 b^{2}-a^{2}\right), \\
p_{1} & =-\frac{m a+1}{2 b}, \\
& =-\frac{m}{2}, \\
p^{2} & \\
p^{2} &
\end{aligned}
$$

(4) Impulse Excitation. For impulse excitation, output function can be written as

$$
C(\psi)=\frac{1}{\sqrt{\beta}} \frac{\alpha \sqrt{\beta} \psi+1}{\left(\psi-\psi_{1}\right)\left(\psi-\psi_{2}\right)\left(\psi-\psi_{3}\right)} .
$$

Three characteristic roots are $\psi_{1}, \psi_{2}, \psi_{3}$, which can be written as

$$
\begin{aligned}
& \psi_{1}=c, \\
& \psi_{2}=a+b i, \\
& \psi_{3}=a-b i .
\end{aligned}
$$

$c$ is real root, $a$ is real part of complex root, and $b$ is the imaginary part of complex root. Response in the time domain can be derived by using inverse Laplace transform method; it can be written as

$$
\begin{aligned}
c(t) & =\frac{1}{\sqrt{\beta}}\left\{-2 E e^{c t}+2 e^{a t}(-F \sin b t+E \cos b t)\right\} \\
p_{1} & =-a+c \\
p_{2} & =b \\
p_{3} & =-a^{2}-b^{2}+a c \\
p_{4} & =b c \\
E & =\frac{m p_{4}-p_{2}}{2\left(p_{1} p_{4}-p_{2} p_{3}\right)} \\
F & =\frac{m p_{3}-p_{1}}{2\left(p_{1} p_{4}-p_{2} p_{3}\right)} \\
m & =\alpha \sqrt{\beta}
\end{aligned}
$$


(5) Harmonic Excitation. For harmonic excitation, output function can be written as

$$
C(\psi)=\frac{1}{\sqrt{\beta}} \frac{\alpha \sqrt{\beta} \psi+1}{\left(\psi^{2}+\omega^{2}\right)\left(\psi-\psi_{1}\right)\left(\psi-\psi_{2}\right)\left(\psi-\psi_{3}\right)} .
$$

Three characteristic roots are $\psi_{1}, \psi_{2}, \psi_{3}$, which can be written as

$$
\begin{aligned}
& \psi_{1}=c, \\
& \psi_{2}=a+b i, \\
& \psi_{3}=a-b i .
\end{aligned}
$$

$c$ is real root, $a$ is real part of complex root, and $b$ is the imaginary part of complex root. Response in the time domain can be derived by using inverse Laplace transform method; it can be written as

$$
\begin{aligned}
& c(t)=\frac{A \omega}{\sqrt{\beta}}\left[\frac{m c+1}{\left[(c-a)^{2}+b^{2}\right]\left(c^{2}+\omega^{2}\right)} e^{c t}\right. \\
& +2 e^{a t}(-F \sin b t+E \cos b t) \\
& +2(N \sin \omega t+M \cos \omega t)] \\
& E=\frac{p_{1} p_{3}+p_{2} p_{4}}{p_{3}^{2}+p_{4}^{2}} \\
& F=\frac{p_{2} p_{3}-p_{1} p_{4}}{p_{3}^{2}+p_{4}^{2}} \\
& M=\frac{p_{5} p_{7}+p_{6} p_{8}}{p_{7}^{2}+p_{8}^{2}} \\
& N=\frac{p_{6} p_{7}-p_{5} p_{8}}{p_{7}^{2}+p_{8}^{2}} \\
& p_{1}=m a+1 \text {, } \\
& p_{2}=m b \text {, } \\
& p_{3}=-2 b^{2}\left(a^{2}-b^{2}+\omega^{2}\right), \\
& p_{4}=-4 a b^{3}+2 b(a-c)\left(a^{2}-b^{2}+\omega^{2}\right), \\
& p_{5}=1 \text {, } \\
& p_{6}=-m \omega \text {, } \\
& p_{7}=-2 \omega^{2}\left(a^{2}+b^{2}-\omega^{2}\right)-4 a c \omega^{2}, \\
& p_{8}=-4 a \omega^{3}+2 \omega c\left(a^{2}+b^{2}-\omega^{2}\right), \\
& m=\alpha \sqrt{\beta} .
\end{aligned}
$$

\subsection{Simulation Results}

(1) Step Excitation. Figures 20-23 are responses in the time domain for step excitation. Lateral axis represents normalized

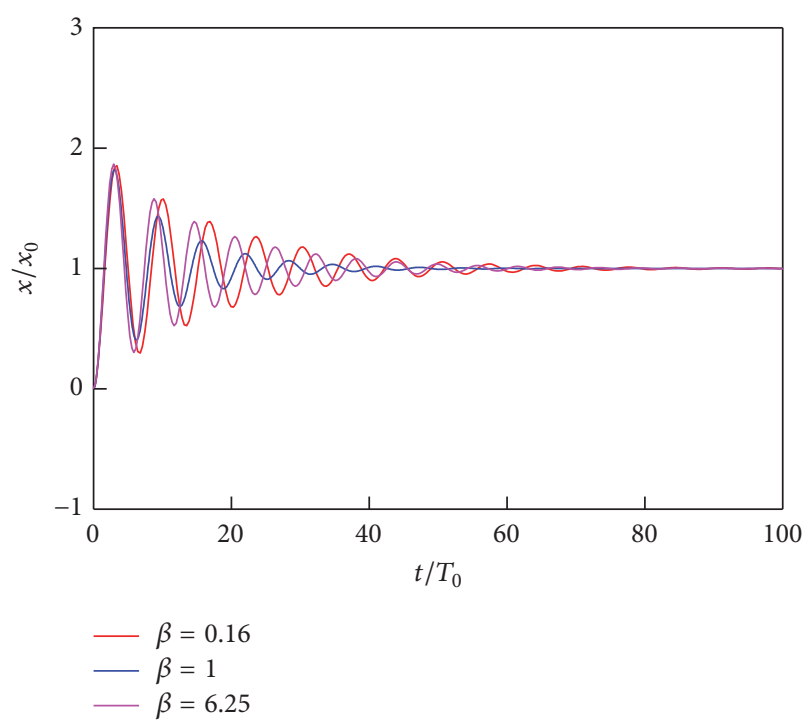

Figure 20: Response in the time domain $(\alpha=1.2, \beta=0.16,1$, 6.25).

time. It can be concluded that the system has the shortest settling time when $\beta=1$ compared with other values of $\beta$. Settling time decreases with the value of $\alpha$.

(2) Slop Excitation. Figures 24-27 are the responses in the time domain for slop excitation; lateral axis represents normalized time. It can be seen from these figures that response in the time domain has a oscillation at the beginning. Duration of oscillation decreases with the value of $\alpha$; then, response is consistent with the input function.

(3) Acceleration Excitation. Figures 28-31 are the responses in the time domain for acceleration excitation. Lateral axis represents normalized time. It can be seen from these figures that response in the time domain is consistent with the input function; this trend has no relationship with the values of $\alpha$ and $\beta$.

(4) Impulse Excitation. Figures 32-35 are the responses in the time domain for impulse excitation. Lateral axis represents normalized time. When the value of $\alpha$ is small, response in the time domain has an obvious oscillation. Oscillation decays with the value of $\alpha$. The system has the shortest settling time when $\beta=1$ compared with the case when $\beta<1$ and $\beta>1$.

4.3. A Comparison Study on the Case When the System Has Optimal Damping and the Case When $\beta=1$. The system has minimum resonance peak when system has optimal damping. It can be proved that all the transmissibility curves will intersect at one point; this point is resonance peak when the system has optimal damping. In this section, a comparison study on the responses in the time domain at two frequencies $\left(\omega / \omega_{0}=2\right.$ and the resonance peak frequency) is made. According to (17), transmissibility can be written as

$$
|G(j \Omega)|=\sqrt{\frac{1+\alpha^{2} \beta \Omega^{2}}{\left(1-\alpha \Omega^{2}\right)^{2}+\beta \Omega^{2}\left(\alpha-\Omega^{2}\right)^{2}}} .
$$




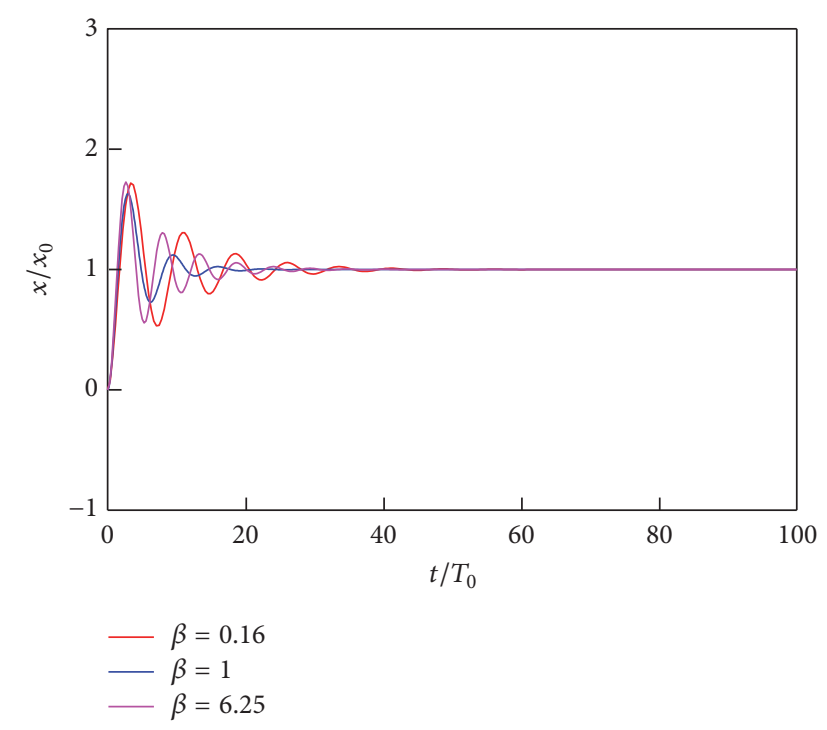

Figure 21: Response in the time domain $(\alpha=1.5, \beta=0.16,1,6.25)$.

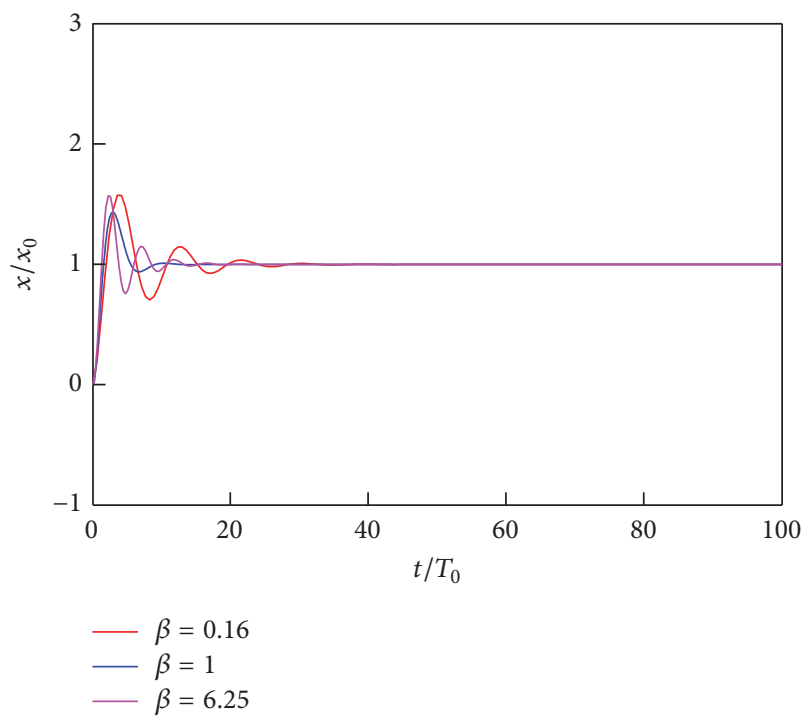

FIgURE 22: Response in the time domain $(\alpha=2, \beta=0.16,1,6.25)$.

$\psi=s / \omega_{0}, s=j \omega$, and, defining $\Omega=\omega / \omega_{0}, \Omega$ is normalized frequency.

$\left|G\left(j \Omega_{1}\right)\right|=\left|G\left(j \Omega_{2}\right)\right|$. Resonance frequency when system has optimal damping can be obtained

$$
\Omega_{0}=\sqrt{\frac{2 \alpha}{1+\alpha^{2}}} .
$$

Taking the derivative of (48) and taking result into (49), the value of $\beta$ can be obtained.

$$
\beta=\frac{1+\alpha^{2}}{2 \alpha}
$$

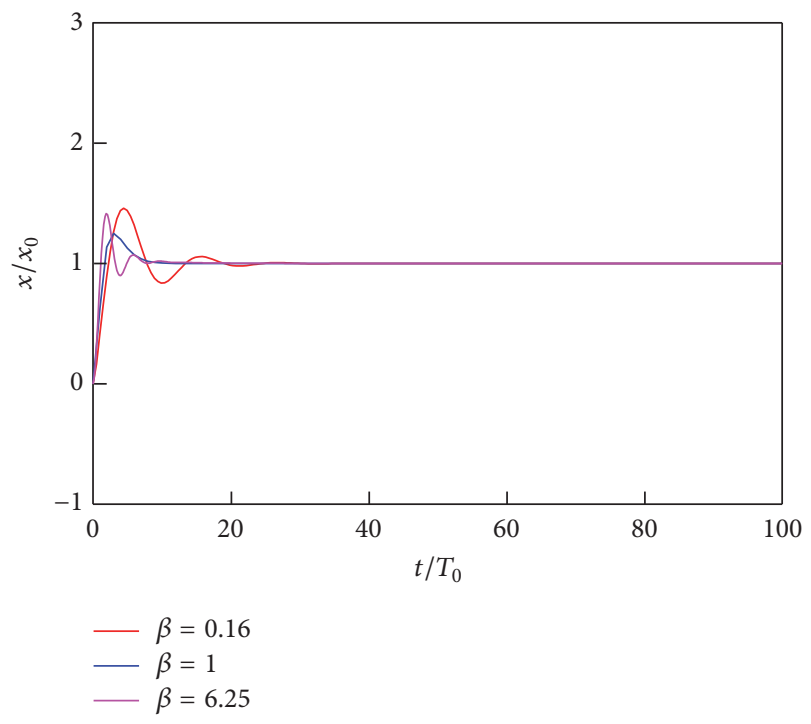

FIgURE 23: Response in the time domain $(\alpha=3, \beta=0.16,1,6.25)$.

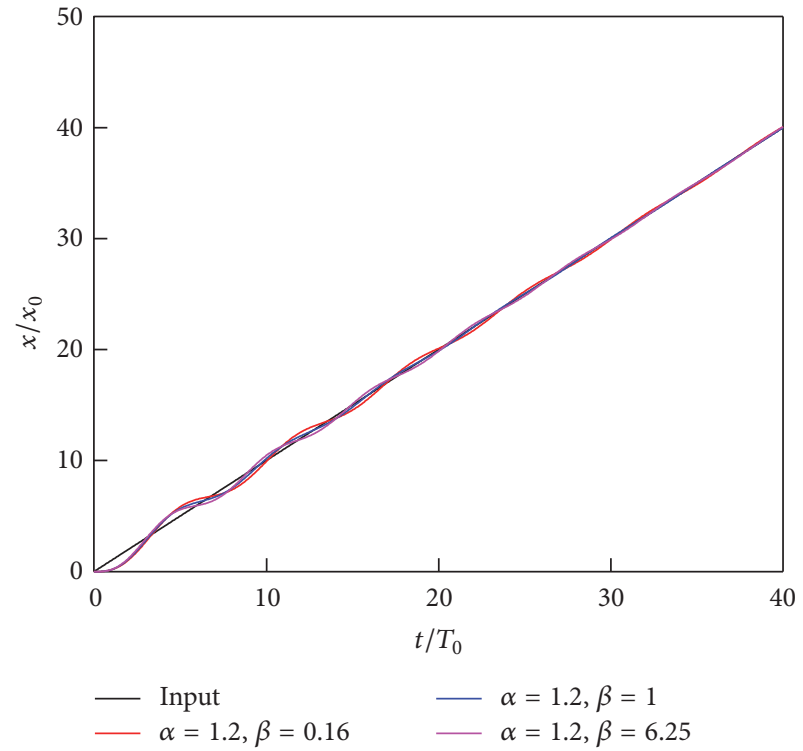

FIGURE 24: Response in the time domain $(\alpha=1.2, \beta=0.16,1$, $6.25)$.

Figures 38-41 are the responses in the time domain for sinusoidal excitation. Two cases are analyzed; the first case is when $\beta=1$ and the second case is when system has optimal damping.

Figure 36 is response in the time domain when $\omega / \omega_{0}=2$ and $\alpha=2$; there are three curves; the black line represents input function, red line represents response in the time domain when $\beta=1$, and blue line represents response in the time domain when system has optimal damping. According to (50), $\beta=1.25$. Longitudinal axis represents displacement transmissibility. It can be seen from the figure that transmissibility when $\beta=1$ is nearly the same as the case when $\beta=1.25$. Figure 37 is response at resonance frequency. 


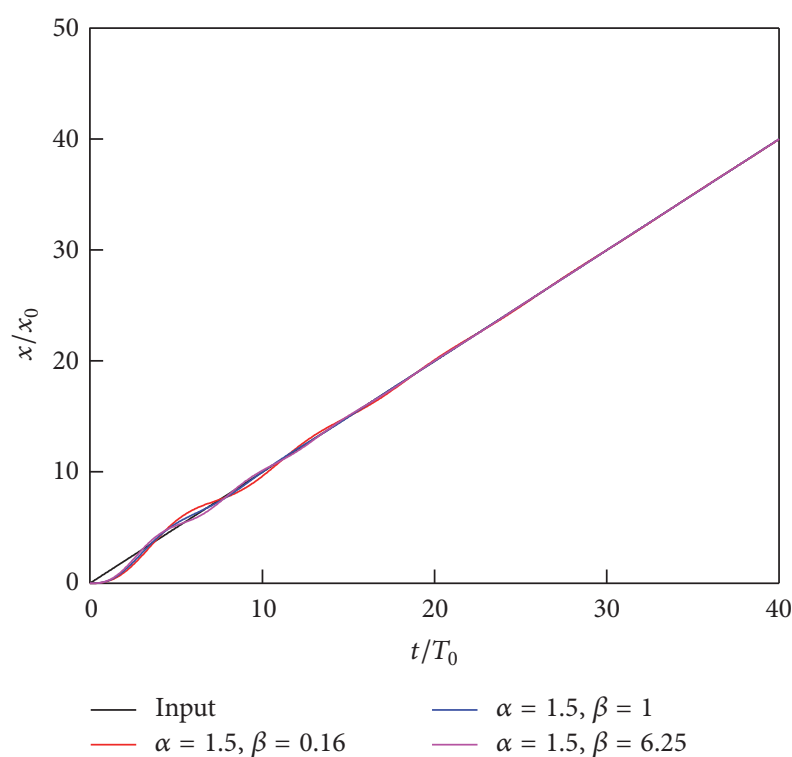

FIGURE 25: Response in the time domain $(\alpha=1.5, \beta=0.16,1$, $6.25)$.

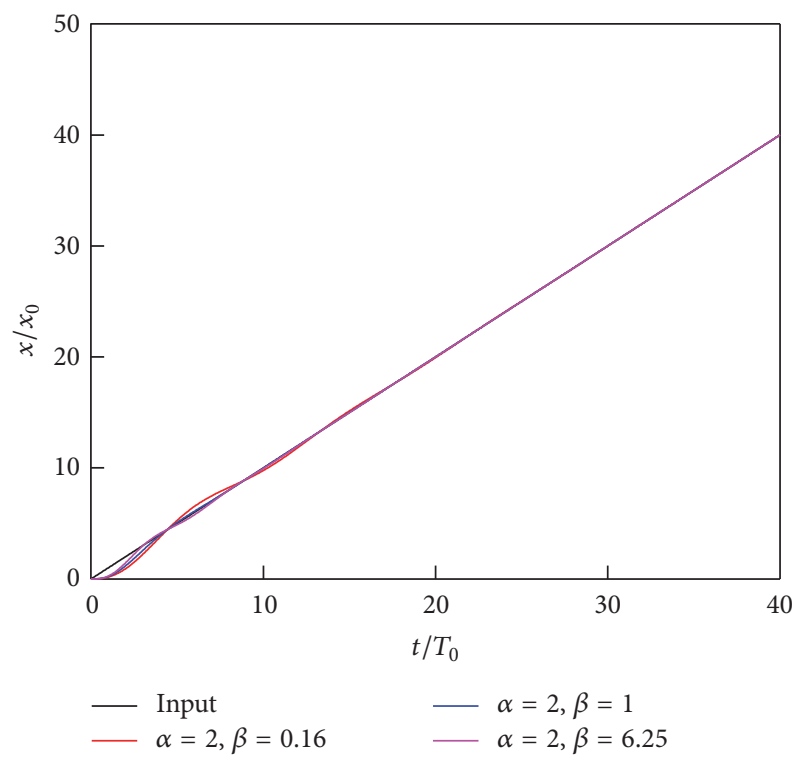

FIGURE 26: Response in the time domain $(\alpha=2, \beta=0.16,1,6.25)$.

It can be seen from the figure that the two cases $(\beta=1$ and $\beta=1.25$ ) nearly have the same transmissibility.

Figure 38 is response in the time domain when $\omega / \omega_{0}=2$ and $\alpha=3$. According to (50), $\beta=1.6667$. It can be seen from the figure that the system has better isolation performance when $\beta=1$ than the case when $\beta=1.6667$. Figure 39 is response at resonance frequency. It can be seen from the figure that the two cases ( $\beta=1$ and $\beta=1.6667)$ nearly have the same transmissibility.

Figure 40 is response in the time domain when $\omega / \omega_{0}=2$ and $\alpha=4$. According to (50), $\beta=2.215$. It can be seen from the figure that the system has better isolation performance

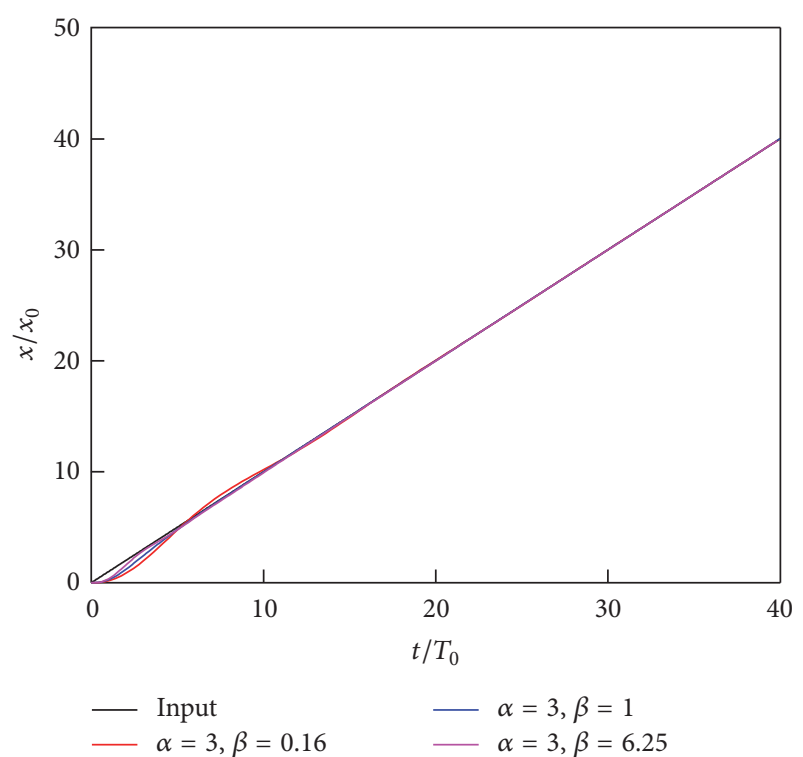

FigURE 27: Response in the time domain $(\alpha=3, \beta=0.16,1,6.25)$.

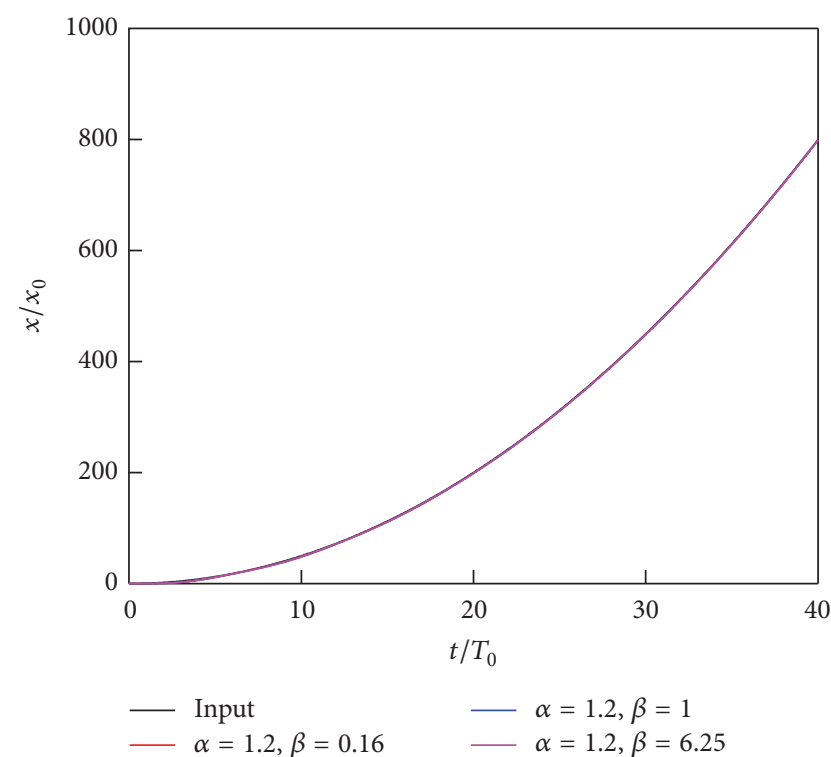

Figure 28: Response in the time domain $(\alpha=1.2, \beta=0.16,1$, 6.25).

when $\beta=1$ than the case when $\beta=2.215$. Figure 41 is response at resonance frequency. It can be seen from the figure that the two cases $(\beta=1$ and $\beta=2.215)$ nearly have the same transmissibility.

With the increasing values of $\alpha$, isolation performance at $\omega / \omega_{0}=2$ becomes better, while the two cases (the case when $\beta=1$ and the case when the system has the optimal damping) nearly have the same transmissibility at resonance frequency.

\section{Transmissibility of Three-Parameter System}

Transmissibility is an important index for isolation performance. In this section, transmissibility of three-parameter 


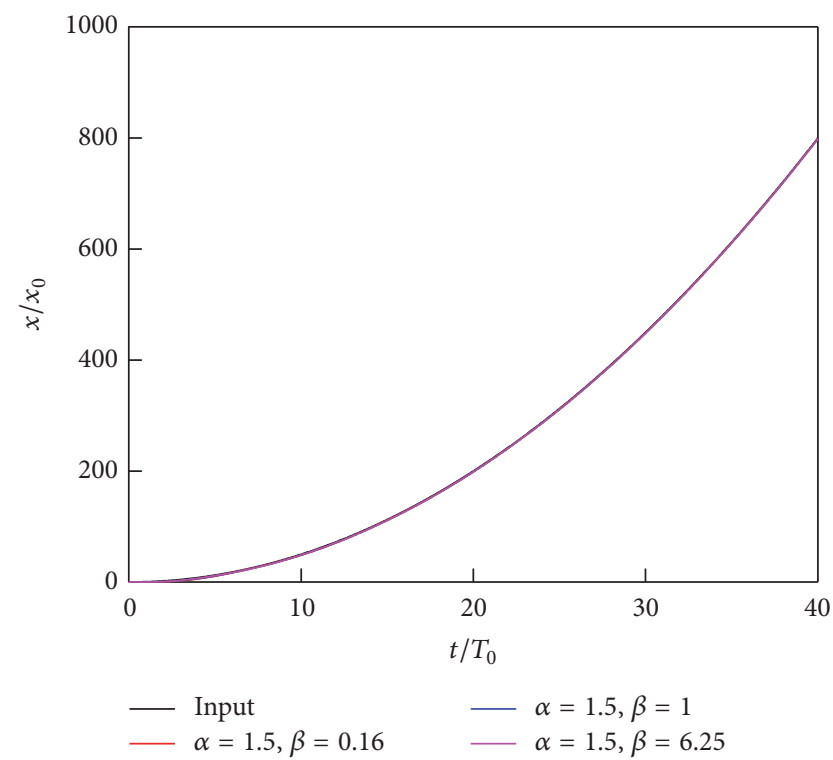

Figure 29: Response in the time domain $(\alpha=1.5, \beta=0.16,1$, $6.25)$.

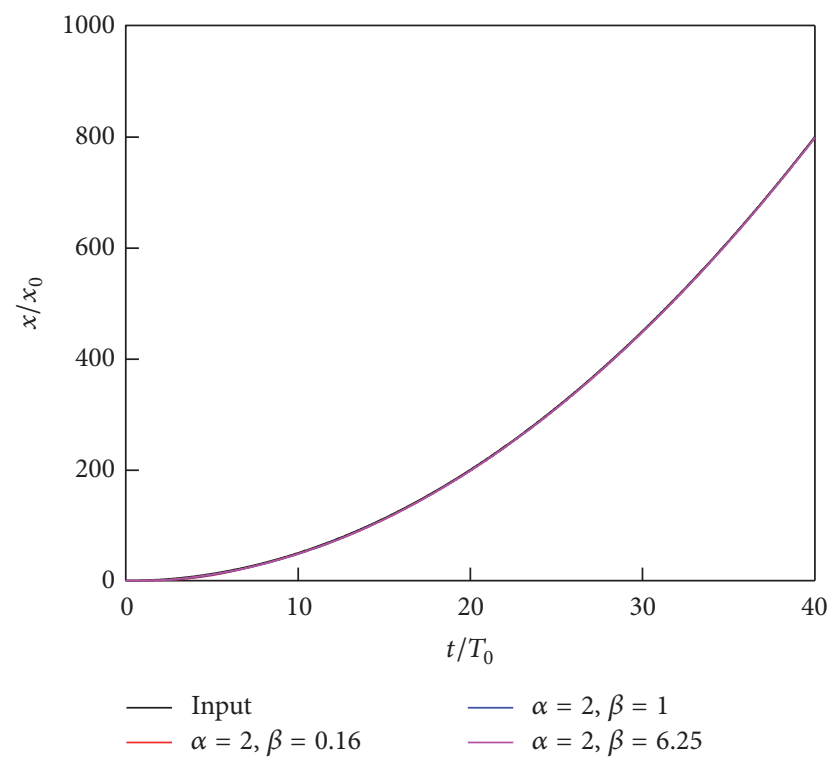

FIGURE 30: Response in the time domain $(\alpha=2, \beta=0.16,1,6.25)$.

system will be discussed. Two cases are analyzed; the first case is when $\beta=1$; the second case is when the system has optimal damping.

Figure 42 is transmissibility of three-parameter system $(\alpha=2)$. Lateral axis represents normalized frequency. Black line is transmissibility for the case when $\beta=1$, resonance frequency is 0.83 , and amplification factor of resonance peak is 1.6823. Red line is transmissibility for the case when system has optimal damping, resonance frequency is 0.89 , and amplification factor of resonance peak is 1.6667. It is shown that amplification factors of two cases are nearly the

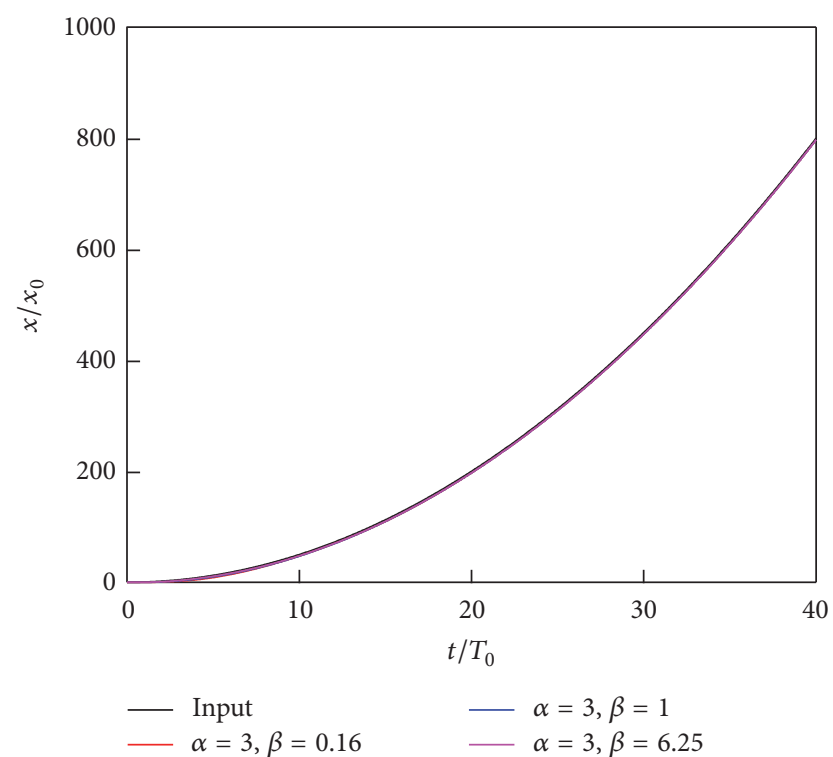

FIGURE 31: Response in the time domain $(\alpha=3, \beta=0.16,1,6.25)$.

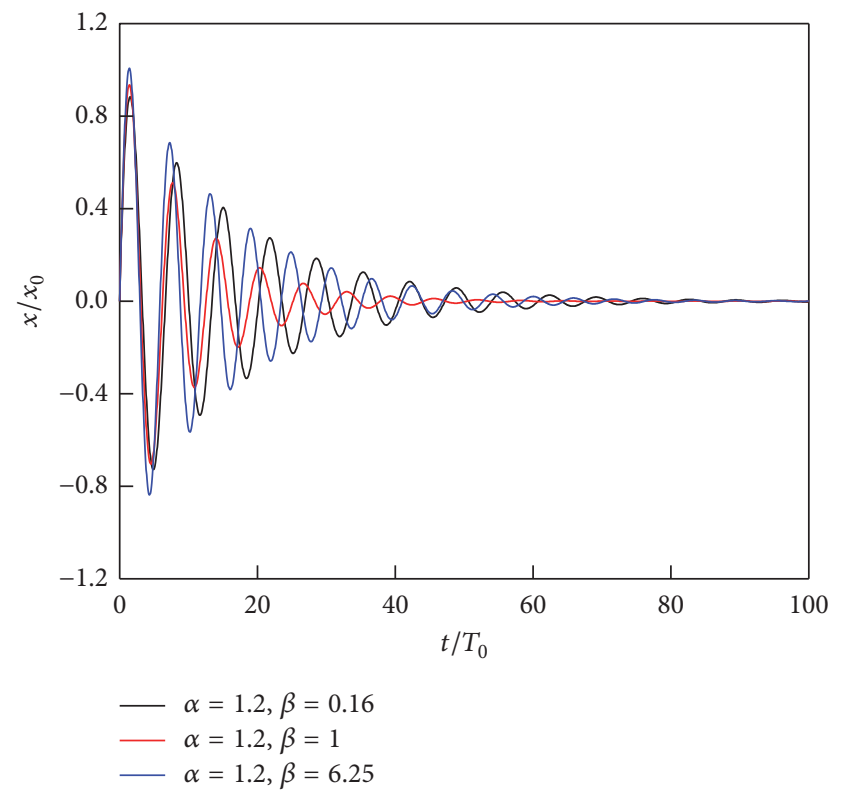

Figure 32: Response in the time domain $(\alpha=1.2, \beta=0.16,1$, $6.25)$.

same (1.6823 and 1.6667). Besides, the two cases nearly have the same transmissibility in the high frequency domain.

Figure 43 is transmissibility of three-parameter system $(\alpha=3)$. Lateral axis represents normalized frequency. Black line is transmissibility for the case when $\beta=1$, resonance frequency is 0.58 , and amplification factor of resonance peak is 1.299. Red line is transmissibility for the case when system has optimal damping, resonance frequency is 0.77 , and amplification factor of resonance peak is 1.25. It is shown that amplification factors of two cases are nearly the same (1.299 and 1.25), while transmissibility characteristics of two 


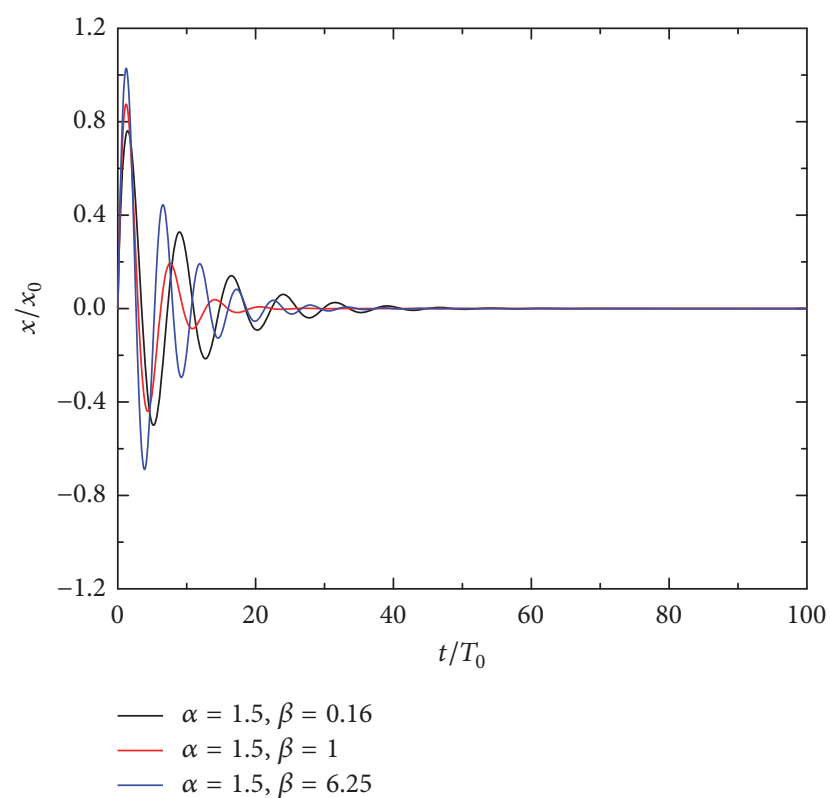

FIGURE 33: Response in the time domain $(\alpha=1.5, \beta=0.16,1$, 6.25).

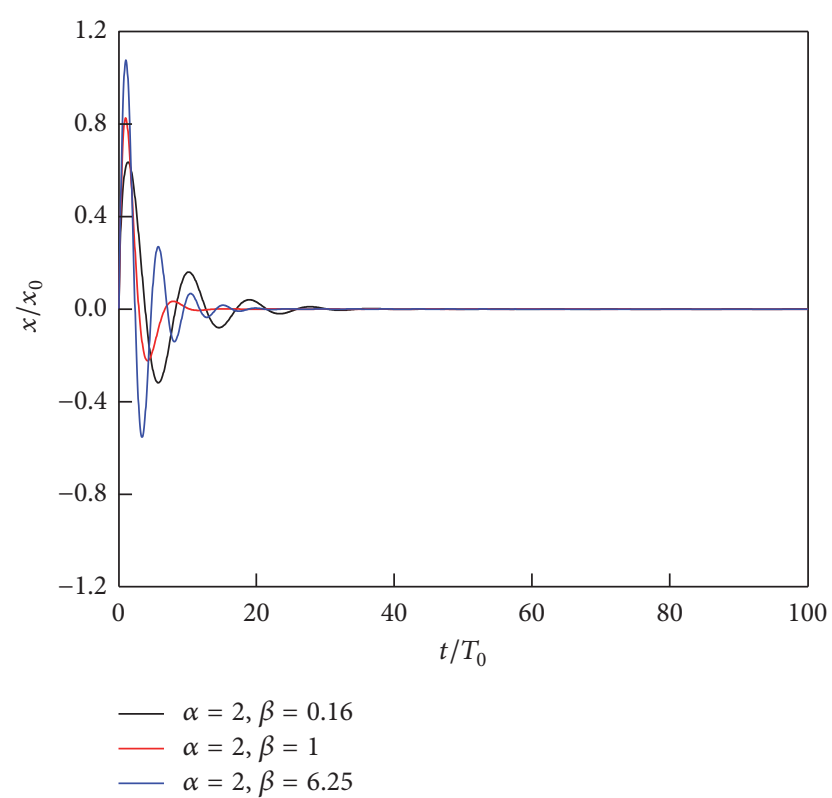

FIGURE 34: Response in the time domain $(\alpha=2, \beta=0.16,1,6.25)$.

cases are different in the high frequency domain. Isolation performance when $\beta=1$ is better than the case when system has optimal damping.

Figure 44 is transmissibility of three-parameter system $(\alpha=4)$. Lateral axis represents normalized frequency. Black line is transmissibility for the case when $\beta=1$, resonance frequency is 0.44 , and amplification factor of resonance peak is 1.1978 . Red line is transmissibility for the case when system has optimal damping, resonance frequency is 0.69 , and amplification factor of resonance peak is 1.3333. It is

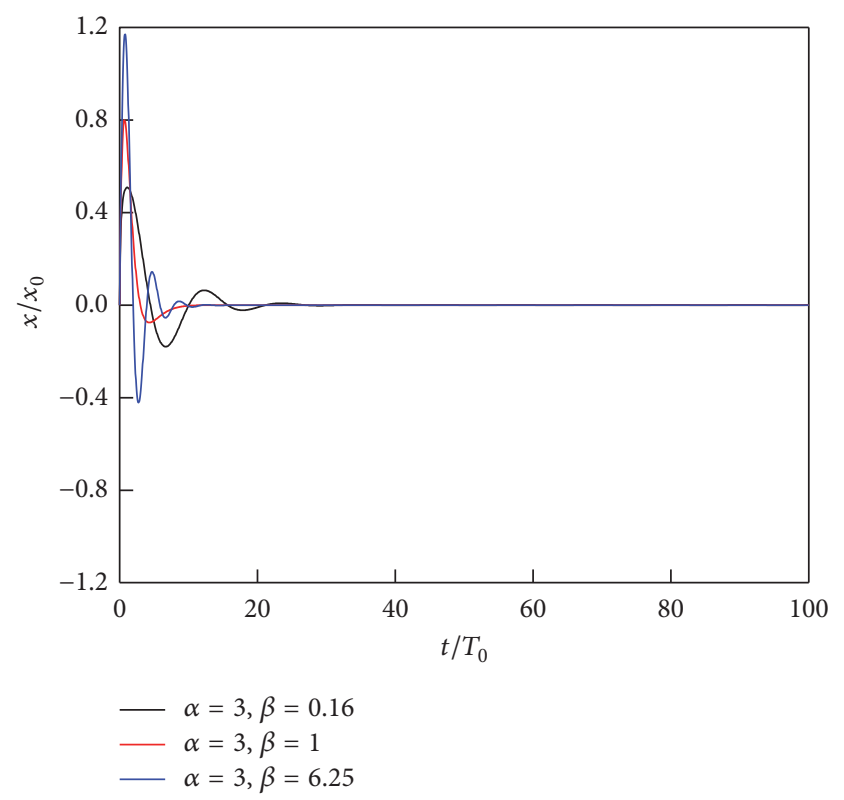

FIgURE 35: Response in the time domain $(\alpha=3, \beta=0.16,1,6.25)$.

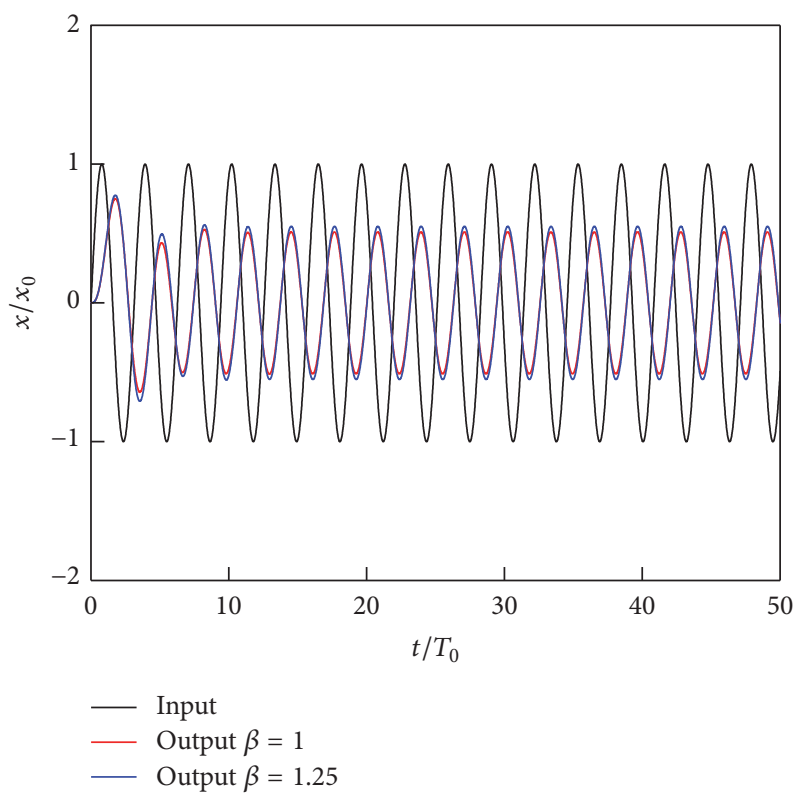

Figure 36: Response in the time domain $(\alpha=2, \beta=1,1.25$, $\left.\omega / \omega_{0}=2\right)$.

shown that amplification factors of two cases are nearly the same (1.1978 and 1.3333), while transmissibility characteristics of two cases are different in the high frequency domain. Isolation performance when $\beta=1$ is better than the case when system has optimal damping.

It can be concluded that the case when $\beta=1$ has better isolation performance in the high frequency domain, but amplification factors of two cases are nearly the same.

Figure 45 is a comparison of resonance factors between the case when $\beta=1$ and the case when system has optimal 


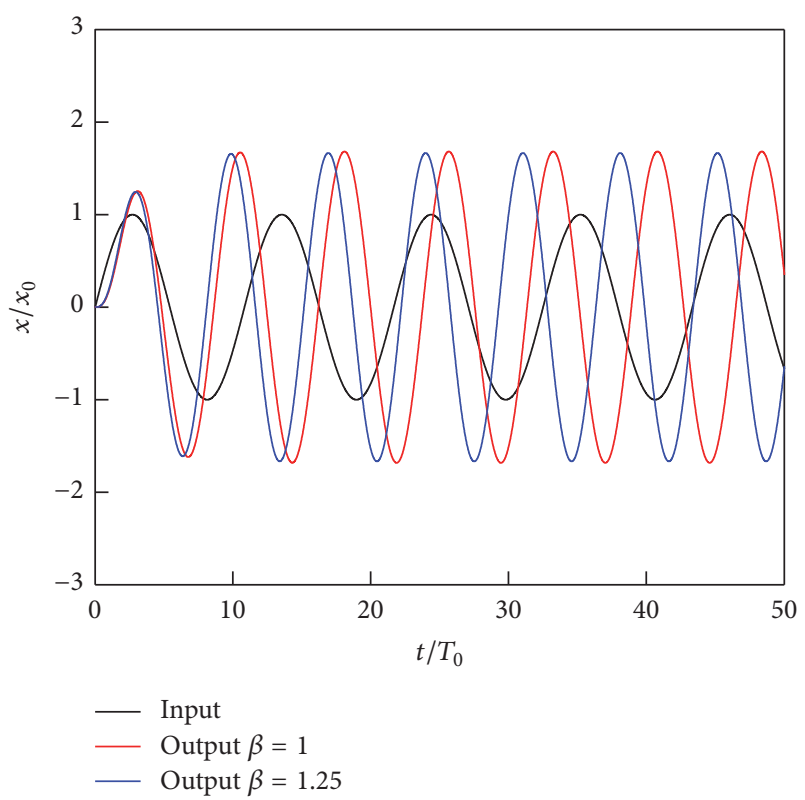

Figure 37: Response in the time domain $(\alpha=2, \beta=1,1.25$, at resonance frequency).

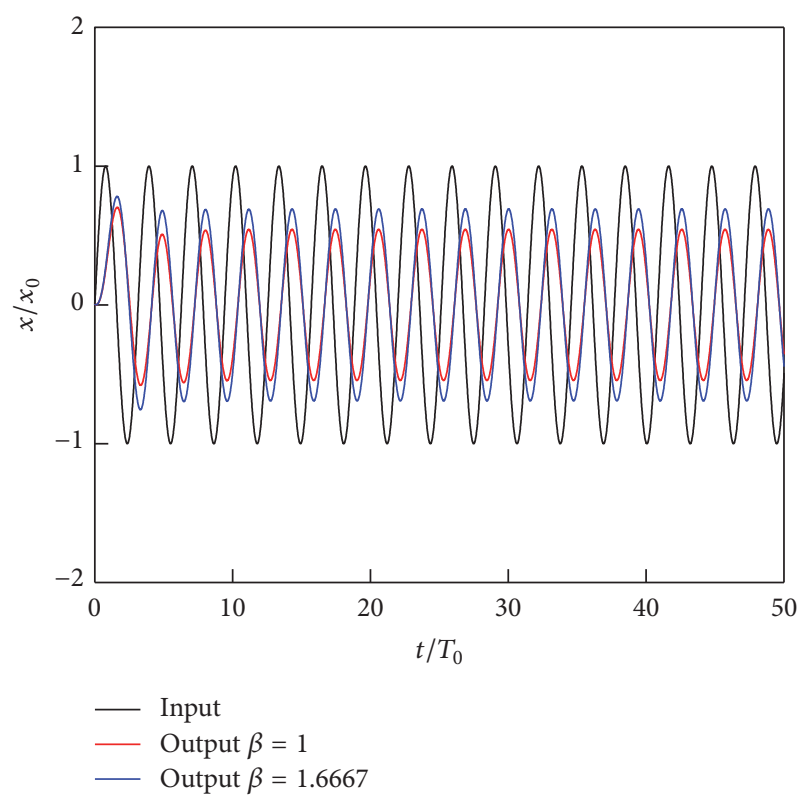

Figure 38: Response in the time domain $(\alpha=3, \beta=1,1.6667$, $\left.\omega / \omega_{0}=2\right)$.

damping. Lateral axis represents the value of $\alpha$. If the value of $\alpha$ is small, there is no difference between these two cases. If the value of $\alpha$ is large, difference between two cases is obvious; the second case has smaller resonance factor than the first case.

Figures $46-50$ are the transmissibility surfaces for different values of $\alpha$ and $\beta$. The first axis represents normalized frequency $\left(\omega / \omega_{0}\right)$, the second axis represents the value of $\alpha$, and the third axis represents transmissibility. Figure 46 is transmissibility surface when $\beta=1$. It can be seen from the

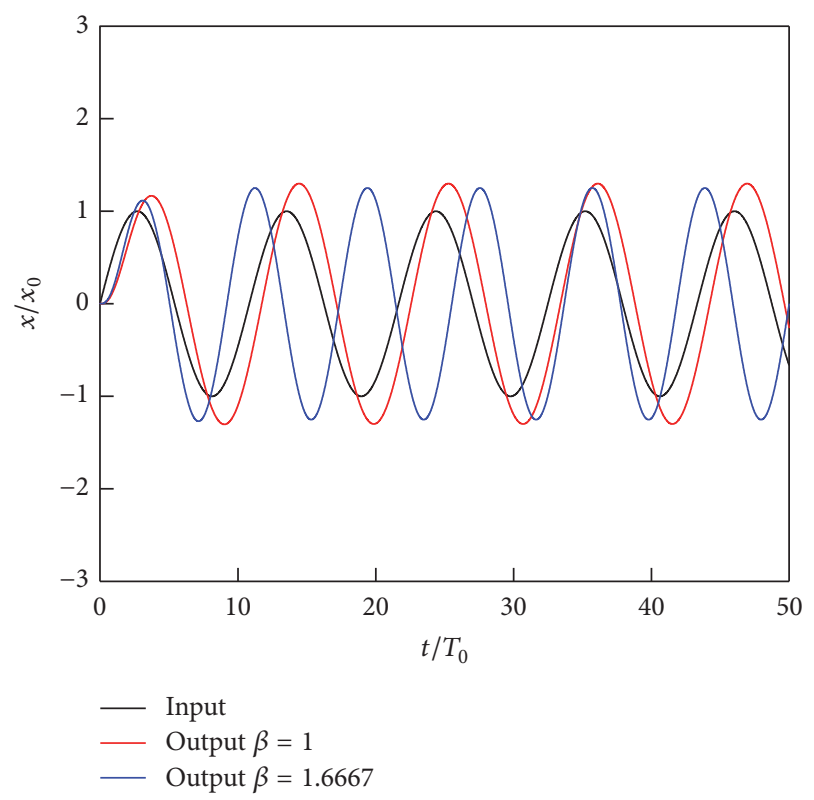

FIGURE 39: Response in the time domain $(\alpha=3, \beta=1,1.6667$, at resonance frequency).

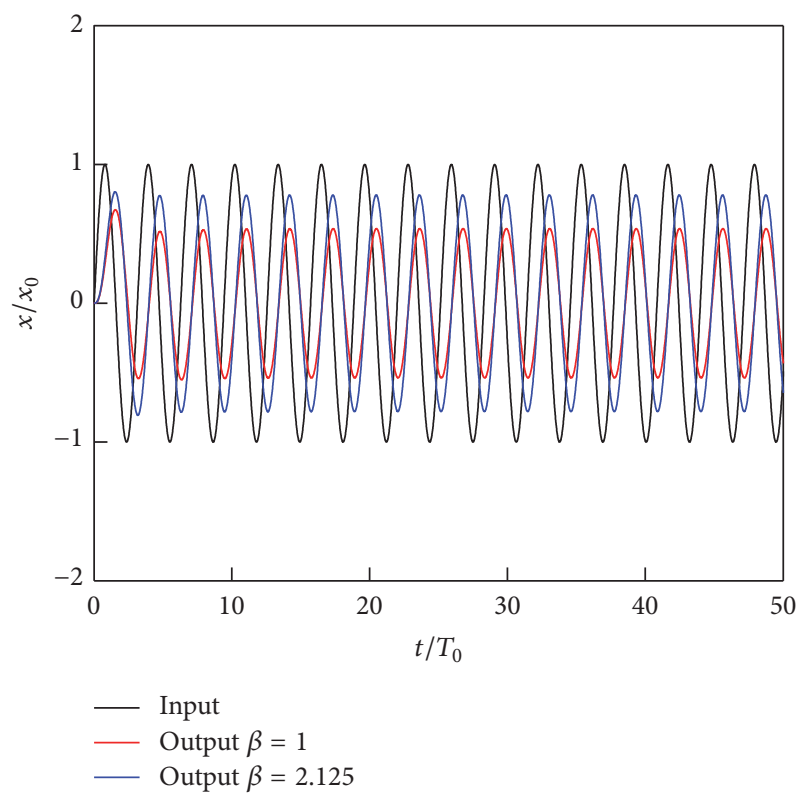

FIGURE 40: Response in the time domain $(\alpha=4, \beta=1,2.125$, $\left.\omega / \omega_{0}=2\right)$.

figure that the largest amplification factor is less than 10 , and amplification factor decreases with the value of $\alpha$. Figure 47 is transmissibility surface when $\alpha=1.2$. It can be seen from the figure that the largest amplification factor is less than 10; with the increasing values of $\beta$, amplification factor decreases, but this trend is not obvious. Figure 48 is transmissibility surface when $\alpha=1.5$. It can be seen from the figure that the largest amplification factor is less than 4; amplification factor decreases obviously compared with the case when $\alpha=1.2$. 


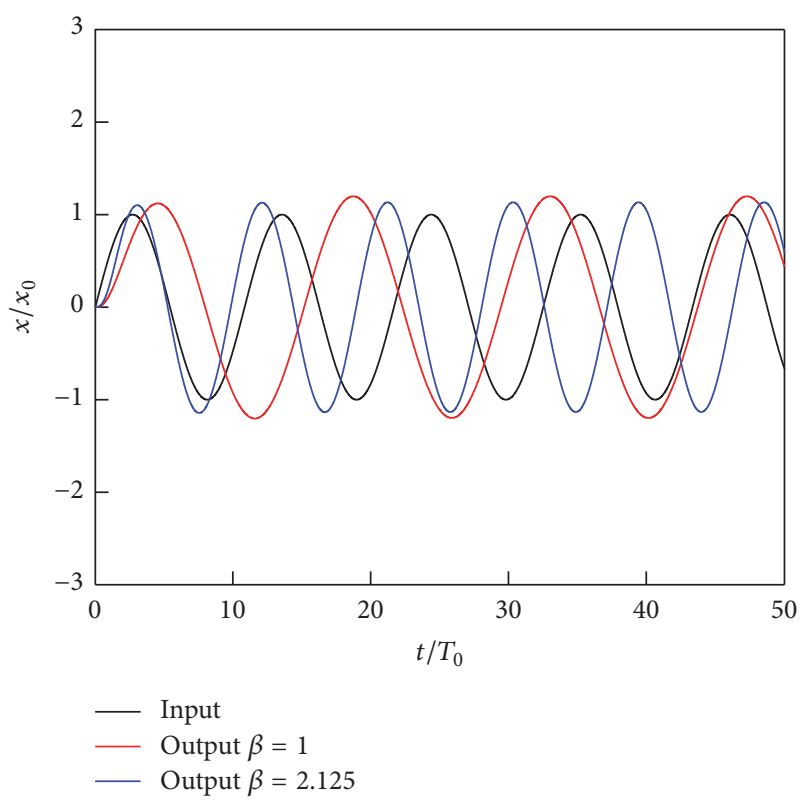

Figure 41: Response in the time domain $(\alpha=4, \beta=1,2.125$, at resonance frequency).

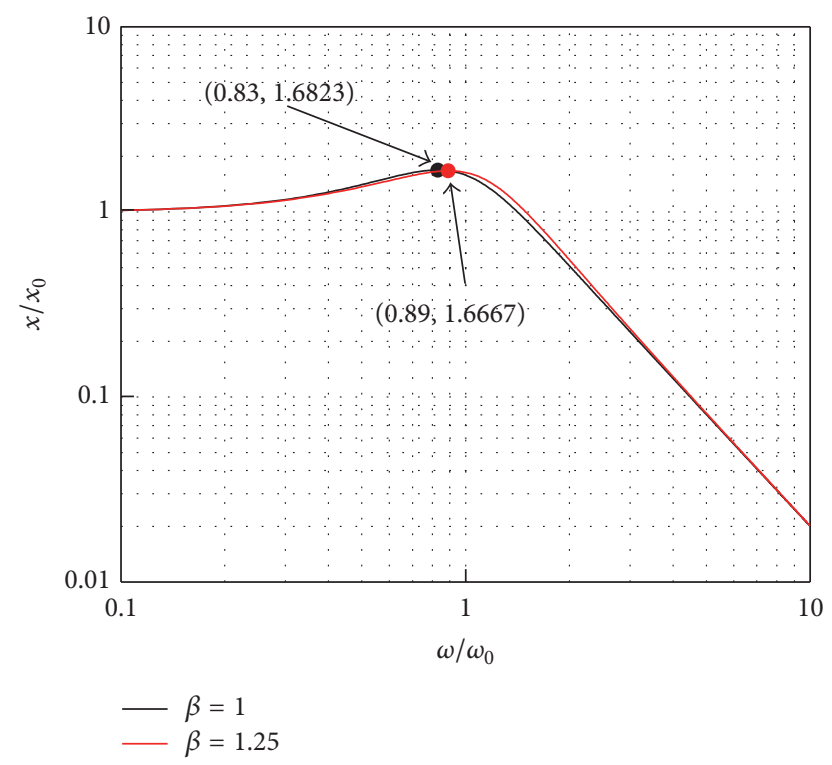

FIgURE 42: Transmissibility $(\alpha=2, \beta=1,1.25)$.

Figure 49 is transmissibility surface when $\alpha=2$. It can be seen from the figure that the largest amplification factor is less than 3; amplification factor decreases obviously compared with the case when $\alpha=1.5$. Figure 50 is transmissibility surface when $\alpha=3$. It can be seen from the figure that the largest amplification factor is less than 3; amplification factor decreases obviously compared with the case when $\alpha=2$. It can be concluded that amplification factor decreases with the value of $\alpha$.

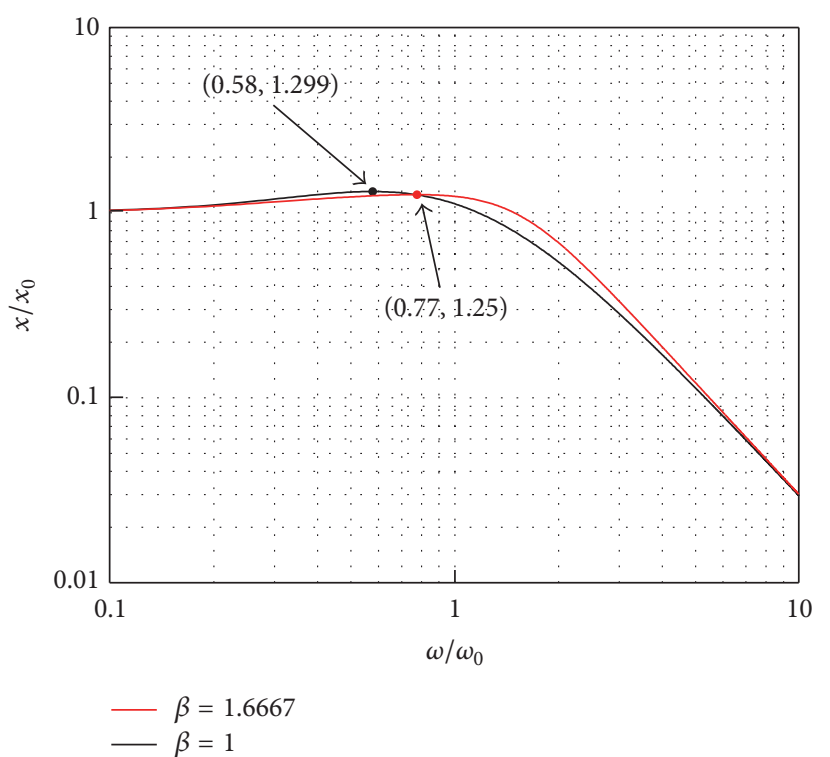

Figure 43: Transmissibility ( $\alpha=3, \beta=1,1.6667)$.

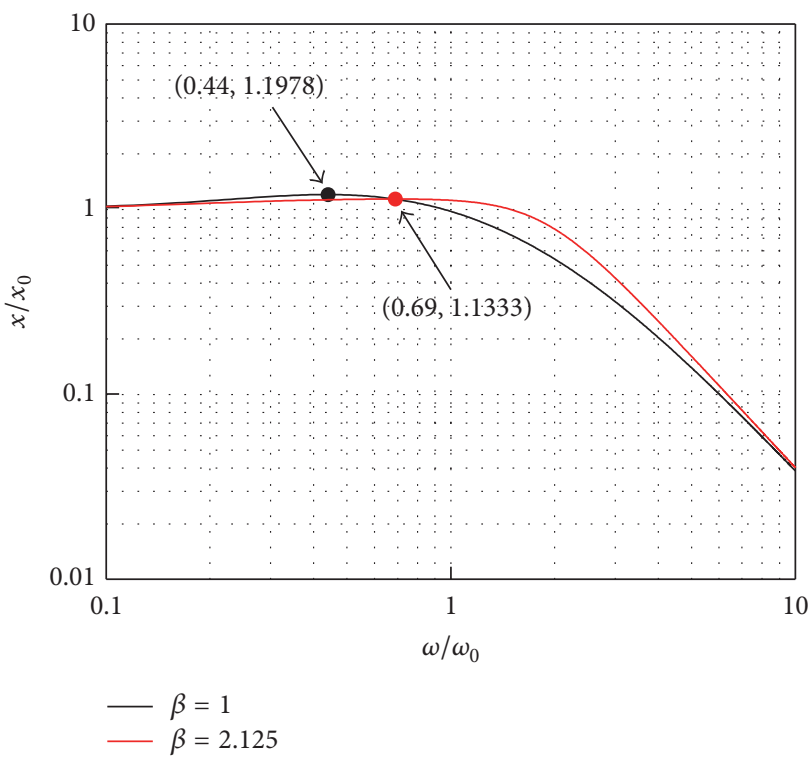

FIGURE 44: Transmissibility $(\alpha=4, \beta=1,2.215)$.

\section{Conclusion}

In this paper, dynamic behavior of three-parameter system has been studied by using normalized model. Different types of excitation have been considered in order to analyze response in the time domain. In order to analyze dynamic behavior in the frequency domain, root locus and phase margin of the system have been analyzed. The results reveal the following:

(1) System under impulse excitation and step excitation has the shortest settling time for the response in the time domain when the value of regulatory factor is 1 ; meanwhile, the system has the largest phase margin. 


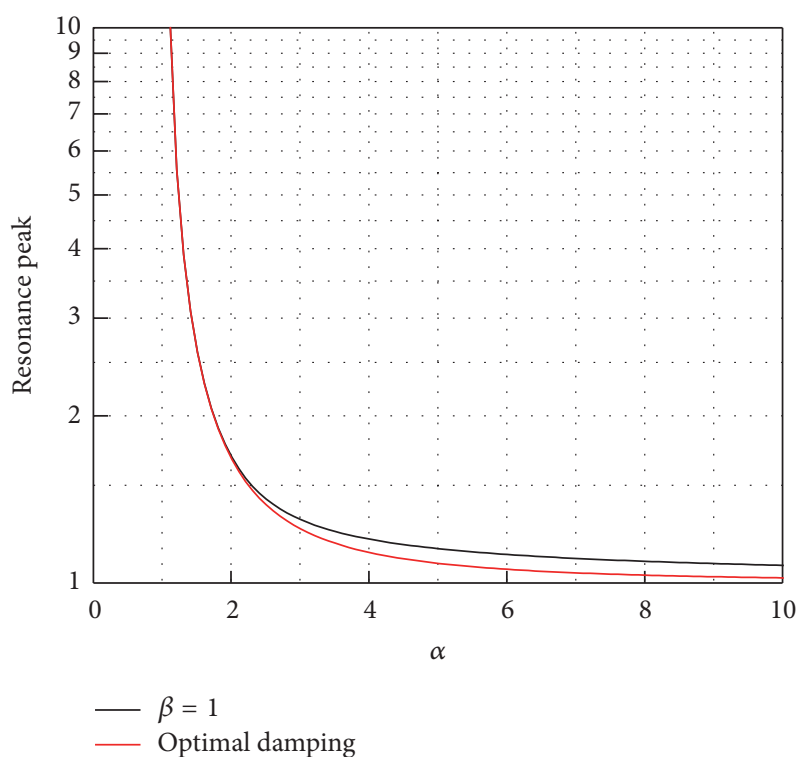

FIGURE 45: Comparison of resonance peaks.

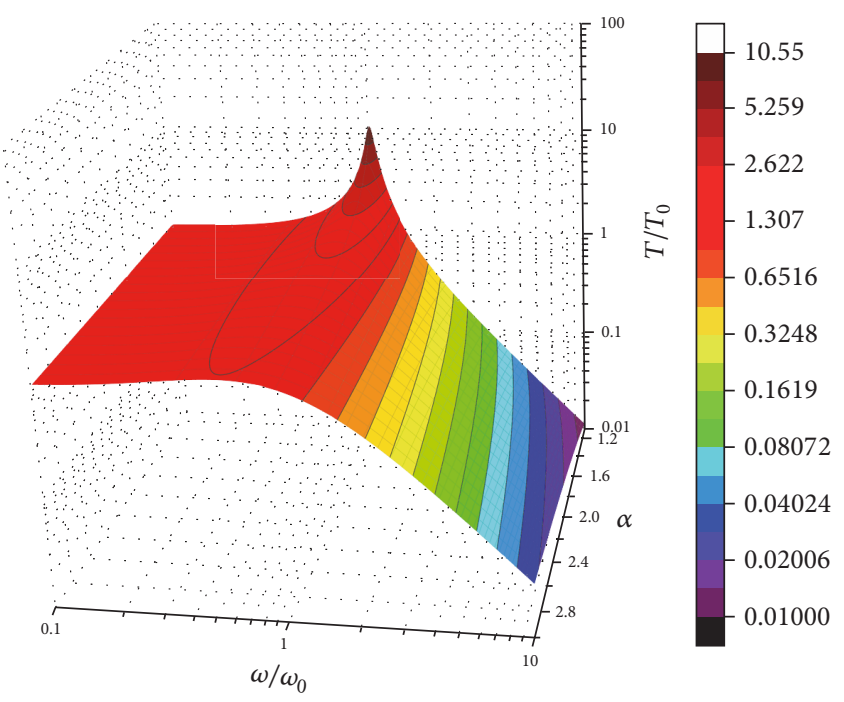

Figure 46: Transmissibility surface $(\beta=1)$.

(2) A comparison study for isolation performance at resonance frequency is made between the case when system has optimal damping and the case when $\beta=$ 1. If stiffness ratio is small, isolation performances of two cases are nearly the same; if stiffness ratio is large, isolation performance of the first case is much better. Previous researches showed that isolation performance of the second case was much better.

(3) Amplification factor decreases with the value of $\alpha$. Because parameter $\alpha$ is the function of stiffness ratio, it means that the system has small amplification factor when stiffness ratio is large.

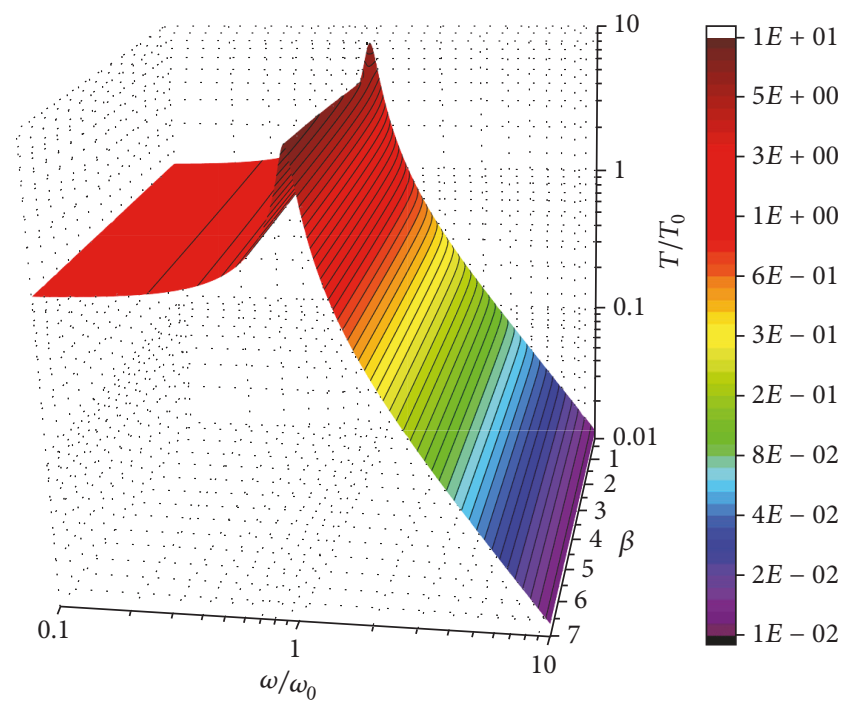

FIgURE 47: Transmissibility surface $(\alpha=1.2, \beta=0.2-7)$.

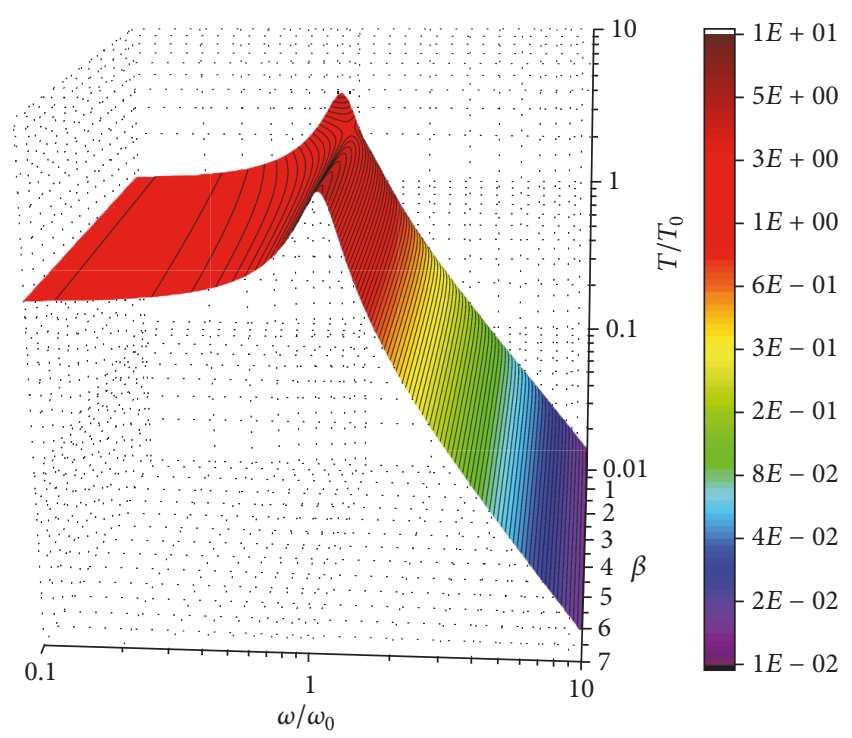

FIgURE 48: Transmissibility surface $(\alpha=1.5, \beta=0.2-7)$.

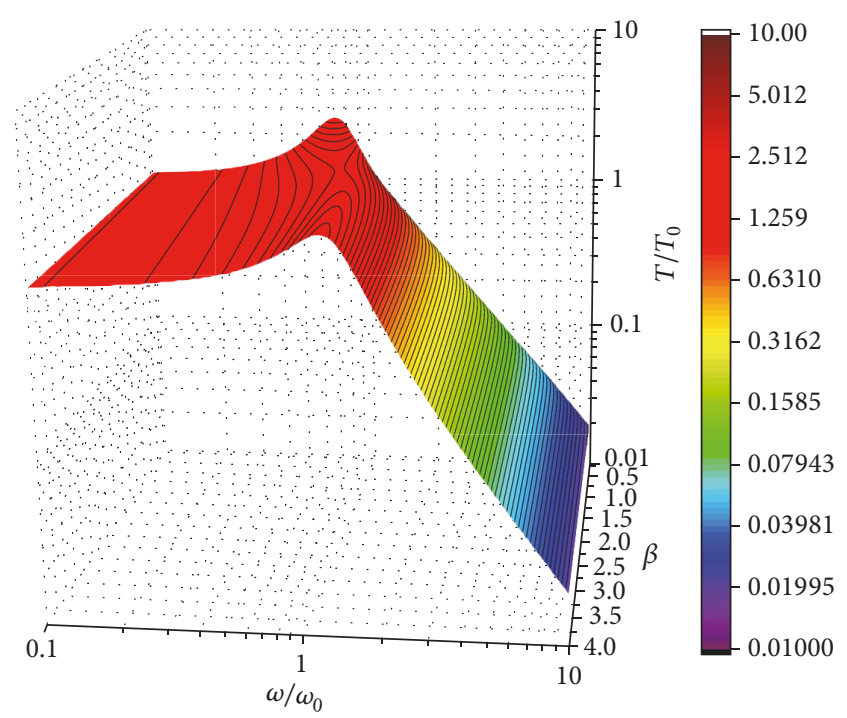

Figure 49: Transmissibility surface $(\alpha=2, \beta=0.2-7)$. 


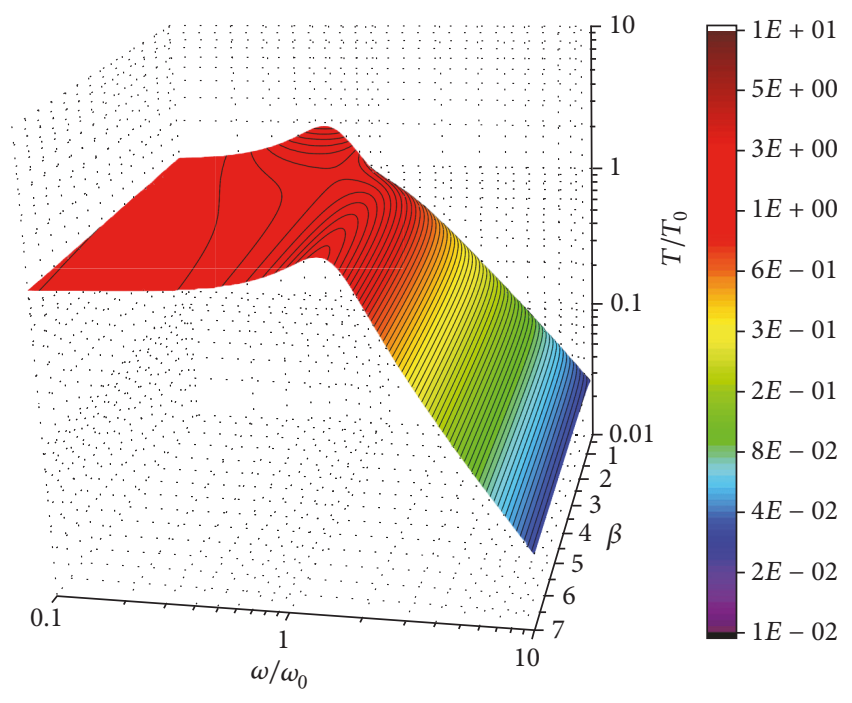

FIGURE 50: Transmissibility surface $(\alpha=3, \beta=0.2-7)$.

(4) The normalized model has two dimensionless parameters $\alpha$ and $\beta$; it can make the dynamic model of threeparameter system more concise than the physical parameter model; therefore, dynamic behavior can be analyzed easily.

\section{Conflicts of Interest}

The authors declare that there are no conflicts of interest regarding the publication of this paper.

\section{Acknowledgments}

This work is supported by the National Basic Research Program of China (Grant no. 2013CB733004) and National Defense Basic Research Plan of China (no. A0320110016).

\section{References}

[1] P. Davis, D. Cunningham, and J. Harrell, "Advanced $1.5 \mathrm{~Hz}$ passive viscous isolation system," Structures, Structural Dynamics, and Materials Conference, vol. 5, pp. 2655-2665, 1994.

[2] B. J. Kawak, "Development of a low-cost, low micro-vibration CMG for small agile satellite applications," Acta Astronautica, vol. 131, pp. 113-122, 2017.

[3] A. Stabile, G. S. Aglietti, G. Richardson, and G. Smet, "A 2collinear-DoF strut with embedded negative-resistance electromagnetic shunt dampers for spacecraft micro-vibration," Smart Materials and Structures, vol. 26, no. 4, Article ID 045031, 2017.

[4] A. Stabile, G. S. Aglietti, G. Richardson, and G. Smet, "Design and verification of a negative resistance electromagnetic shunt damper for spacecraft micro-vibration," Journal of Sound and Vibration, vol. 386, pp. 38-49, 2017.

[5] A. J. Bronowicki, "Vibration isolator for large space telescopes," Journal of Spacecraft and Rockets, vol. 43, no. 1, pp. 45-53, 2006.

[6] J. F. Yang, Z.-B. Xu, Q.-W. Wu, Z.-S. Wang, H. Li, and S. He, "Design of a vibration isolation system for the space telescope,"
Journal of Guidance, Control, and Dynamics, vol. 38, no. 12, pp. 2441-2448, 2015.

[7] R. E. Erwin, V. Babuska, and L. Sullivan, "On-orbit active vibration isolation: the Satellite Ultraquiet Isolation Technologies Experiment (SUITE)," AIAA Space Conference and Exposition, vol. 13, no. 3, 10 pages, 2003.

[8] Z.-J. Wei, D.-X. Li, Q. Luo, and J.-P. Jiang, "Modeling and analysis of a flywheel microvibration isolation system for spacecrafts," Advances in Space Research, vol. 55, no. 2, pp. 761777, 2015.

[9] V. Preda, J. Cieslak, D. Henry, S. Bennani, and A. Falcoz, "Microvibration attenuation based on Ho/LPV theory for high stability space missions," Journal of Physics: Conference Series, vol. 659, no. 1, Article ID 012026, 2015.

[10] D. Kamesh, R. Pandiyan, and A. Ghosal, "Modeling, design and analysis of low frequency platform for attenuating microvibration in spacecraft," Journal of Sound and Vibration, vol. 329, no. 17, pp. 3431-3450, 2010.

[11] M. Remedia, G. S. Aglietti, G. Richardson, and M. Sweeting, "Integrated semiempirical methodology for microvibration prediction," AIAA Journal, vol. 53, no. 5, pp. 1236-1250, 2015.

[12] Y. Zhang, M. Li, Z.-Y. Song, J.-J. Shan, X. Guan, and L. Tang, "Design and analysis of a moment control unit for agile satellite with high attitude stability requirement," Acta Astronautica, vol. 122, pp. 90-105, 2016.

[13] J. E. Ruzicka and T. F. Derby, "Influence of damping in vibration isolation," Shock and Vibration Information Center, 1971.

[14] I. Yamakawa, "Free and transient vibration of an elastically supported damper system," 1961.

[15] L. P. Davis, D. Cunningham, A. S. Bicos, M. Enright, and C. D. Johnson, "Adaptable passive viscous damper: an adaptable DStrutTM," Smart Structures and Materials 1994: Passive Damping, vol. 2193, pp. 47-58, 1994.

[16] L. P. Davis, D. R. Carter, T. T. Hyde, and C. D. Johnson, "Second-generation hybrid D-strut," in Proceedings of the Smart Structures \& Materials '95, pp. 161-175, San Diego, CA.

[17] L. P. Davis, D. C. Cunningham, and D. H. Duncan, "Three parameter viscous damper and isolator," United States Patent, 1994.

[18] D. Cunningham and P. Davis, "A multi axis passive isolation system for a magnetic bearing reaction wheel," Advances in theAstronautical Sciences, vol. 95, no. Article ID 80426, 1993.

[19] J. J. Rodden, H. J. Dougherty, and L. F. Reschke, "ine of sight performance improvement with reaction-wheel isolation," Spacecraft Design, Testing and Performance, Article ID $19870045457,1987$.

[20] J. Tosovsky, V. Janulik, and D. T. Ruebsamen, "Adaptive three parameter isolator assemblies including external magnetorheological valves," European Patent, 2016.

[21] R. Goold and Z. Rogers, "Low profile three parameter isolators and isolation systems employing the same," United States Patent, 2016.

[22] D. W. Smith and S. Hadden, Thermally-conductive vibration isolators and spacecraft isolation systems employing the same, United States Patent, 2014.

[23] W. L. Meacham, E. Bridges, and A. Margolis, Three parameter damper anisotropic vibration isolation mounting assembly, United States Patent, 2016.

[24] D. T. Ruebsamen, J. H. Boyd, and T. S. Davis, Vibration isolation apparatus and methods of manufacture, United States Patent, 2011. 
[25] D. Martinez, J. Pagan, and R. Goold, Isolators including damper assemblies having variable annuli and spacecraft isolation systems employing the same, United States Patent, 2014.

[26] D. C. Cunningham, Isolators including damper assemblies having variable annuli and spacecraft isolation systems employing the same, European Patent, 1994.

[27] S. Hadden and D. T. Ruebsamen, Three parameter, multi-axis isolators, isolation systems employing the same, and methods for producing the same, European Patent, and methods for producing the same, 2012.

[28] M. J. Brennan, A. Carrella, T. P. Waters, and V. Lopes Jr., "On the dynamic behaviour of a mass supported by a parallel combination of a spring and an elastically connected damper," Journal of Sound and Vibration, vol. 309, no. 3-5, pp. 823-837, 2008.

[29] L.-K. Liu, G.-T. Zheng, and W.-H. Huang, "Study of liquid viscosity dampers in octo-strut platform for whole-spacecraft vibration isolation," Acta Astronautica, vol. 58, no. 10, pp. 515522, 2006.

[30] Y. Zhang, J.-R. Zhang, and S.-J. Xu, "Parameters design of vibration isolation platform for control moment gyroscopes," Acta Astronautica, vol. 81, no. 2, pp. 645-659, 2012.

[31] Y. Zhang and J.-R. Zhang, "Analysis of influence of vibration isolation platform on attitude control system and its parameter selection," Yuhang Xuebao/Journal of Astronautics, vol. 34, no. 5, pp. 657-664, 2013.

[32] J. Wang and S. G. Zhao, "A test method of dynamic parameters of vibration isolators," Journal of Vibration Engineering, vol. 27, no. 6, pp. 885-892, 2014.

[33] J. Wang, S.-G. Zhao, D.-F. Wu, and M. Luo, "A test method of dynamic damping coefficient of micro-vibration isolators," Hangkong Xuebao/Acta Aeronautica et Astronautica Sinica, vol. 35, no. 2, pp. 454-460, 2014.

[34] C.-X. Wang, J.-Y. Sun, Z.-Y. Zhang, and H.-X. Hua, “Design and experiment of a three-parameter isolation system with optimal damping," Jixie Gongcheng Xuebao/Journal of Mechanical Engineering, vol. 51, no. 15, pp. 90-96, 2015.

[35] J. Wang, S.-G. Zhao, and D.-F. Wu, "Performance of a type of nonlinear fluid microvibration isolators," Journal of Aerospace Engineering, vol. 28, no. 6, p. 04015002, 2015.

[36] X. Wang, H.-X. Yao, and G.-T. Zheng, "Enhancing the isolation performance by a nonlinear secondary spring in the Zener model," Nonlinear Dynamics, vol. 87, no. 4, pp. 2483-2495, 2017.

[37] W.-K. Shi, C. Qian, Z.-Y. Chen, Y. Cao, and H.-H. Zhang, "Modeling and Dynamic Properties of a Four-Parameter Zener Model Vibration Isolator," Shock and Vibration, vol. 2016, Article ID 5081812, 2016. 


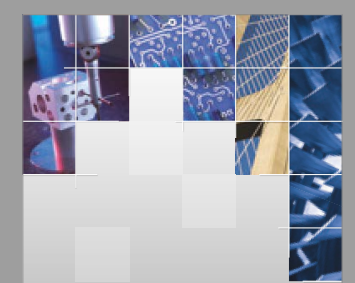

\section{Enfincering}
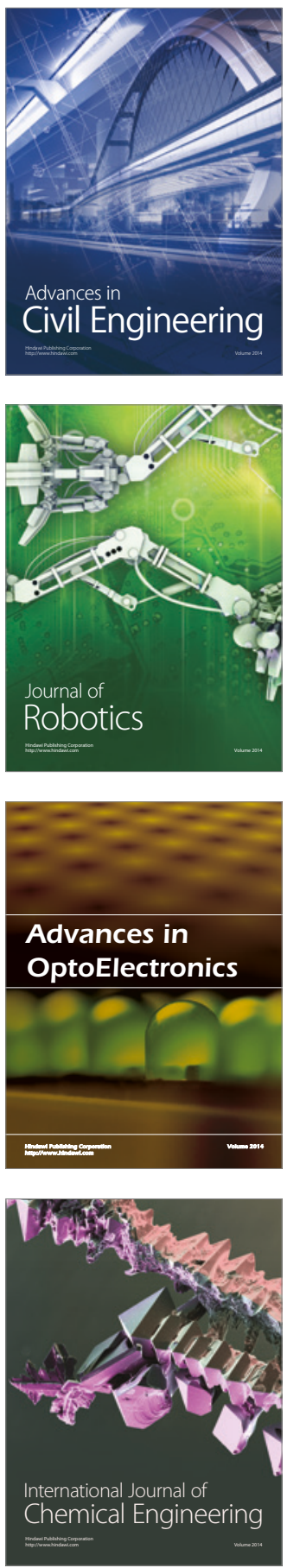

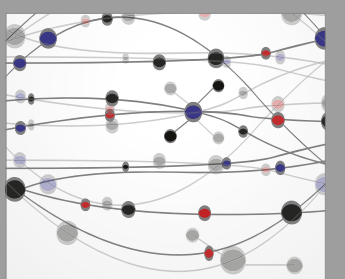

The Scientific World Journal

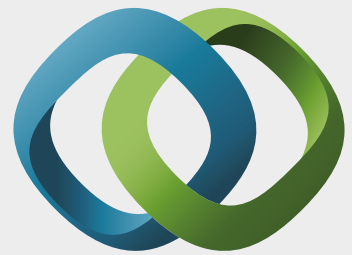

\section{Hindawi}

Submit your manuscripts at

https://www.hindawi.com
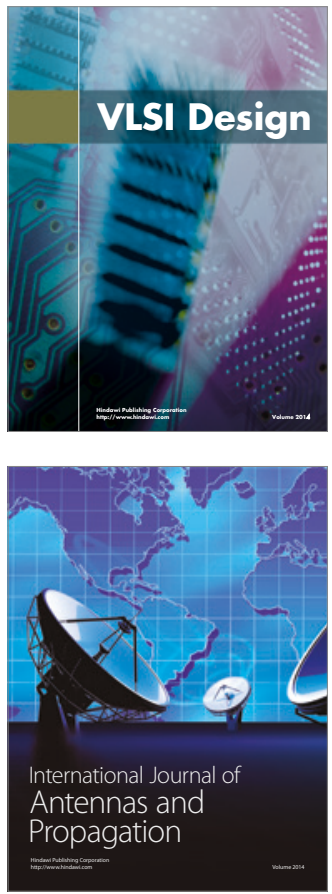

\section{Rotating}

Machinery
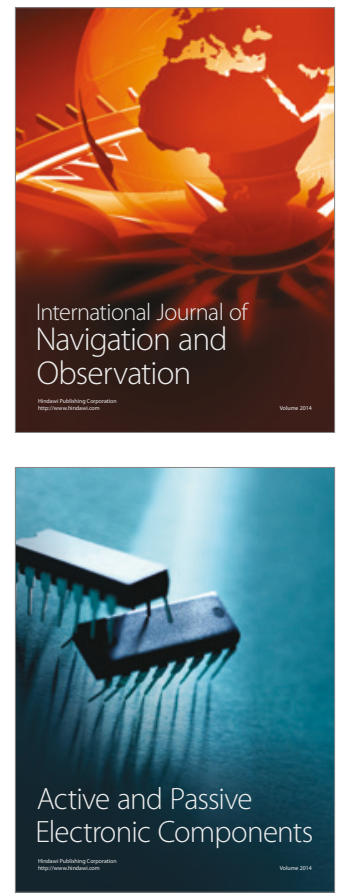
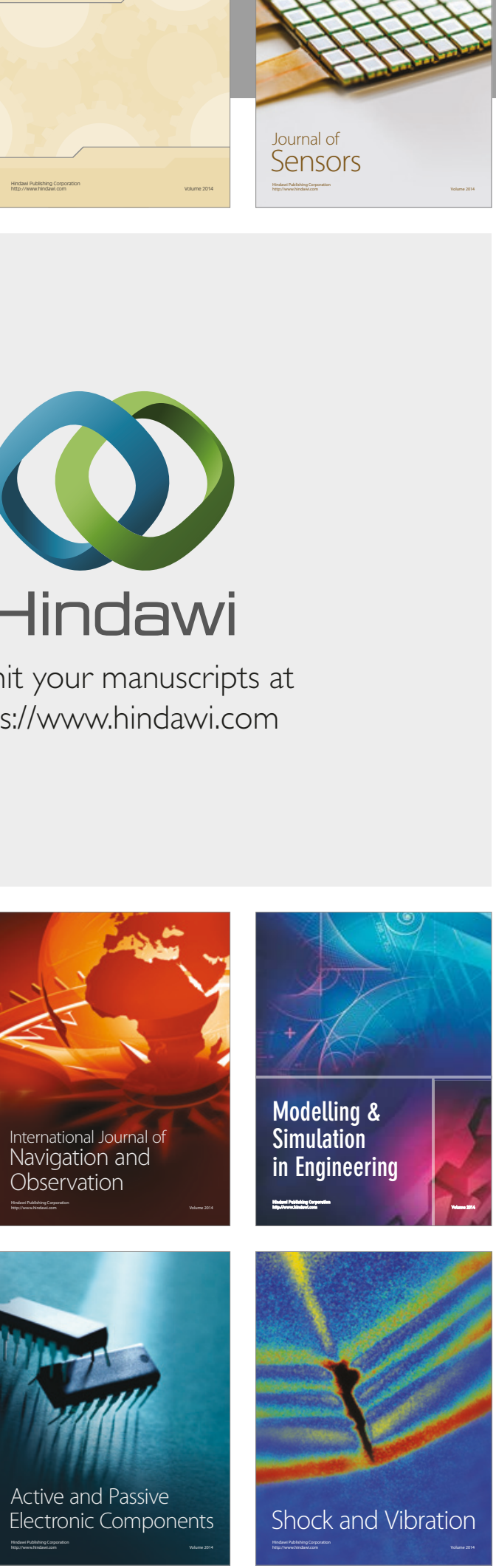
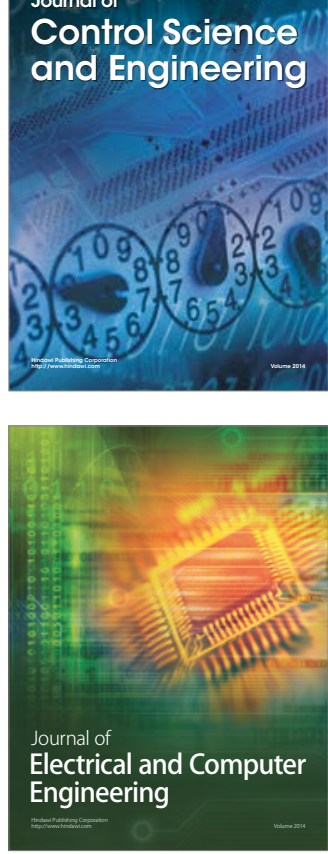

Distributed

Journal of

Control Science

and Engineering
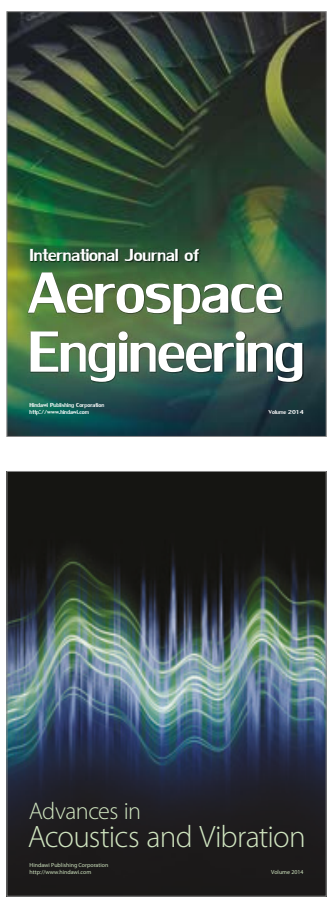

Sensor Networks 